Draft Version May 27, 2021

Typeset using LATEX default style in AASTeX63

\title{
Spectral Observations of Superthin Galaxies
}

\author{
Dmitry Bizyaev,${ }^{1,2,3}$ D. I. Makarov,${ }^{3}$ V. P. Reshetnikov, ${ }^{4,3}$ A. V. Mosenkov ${ }^{5}$ S. J. Kautsch,${ }^{6}$ and \\ A. V. Antipova ${ }^{3}$ \\ ${ }^{1}$ Apache Point Observatory and New Mexico State University, Sunspot, NM, 88349, USA \\ ${ }^{2}$ Sternberg Astronomical Institute, Moscow State University, Universitetskiy prosp. 13, 119992, Moscow, Russia \\ ${ }^{3}$ Special Astrophysical Observatory, Russian Academy of Sciences, 369167 Nizhnij Arkhyz, Russia \\ ${ }^{4}$ St.Petersburg State University, 7/9 Universitetskaya nab., St.Petersburg, 199034 Russia \\ ${ }^{5}$ Central Astronomical Observatory, Russian Academy of Sciences, 65/1 Pulkovskoye chaussee, St.Petersburg, 196140 Russia \\ ${ }^{6}$ Nova Southeastern University, Fort Lauderdale, FL, 33314, USA
}

(Received June 00, 0000; Revised June 00, 0000; Accepted May 27, 2021)

\begin{abstract}
We conduct spectral observations of 138 superthin galaxies (STGs) with high radial-to-vertical stellar disk scales ratio with the Dual Imaging Spectrograph (DIS) on the $3.5 \mathrm{~m}$ telescope at the Apache Point Observatory (APO) to obtain the ionized gas rotation curves with $\mathrm{R} \sim 5000$ resolution. We also performed near infrared (NIR) $\mathrm{H}$ and Ks photometry for 18 galaxies with the NICFPS camera on the $3.5 \mathrm{~m}$ telescope. The spectra, the NIR photometry and published optical and NIR photometry are used for modeling that utilizes the thickness of the stellar disk and rotation curves simultaneously. The projection and dust extinction effects are taken into account. We evaluate eight models that differ by their free parameters and constraints. As a result, we estimated masses and scale lengths of the galactic dark halos. We find systematic differences between the properties of our red and blue STGs. The blue STGs have a large fraction of dynamically under-evolved galaxies whose vertical velocity dispersion is low in both gas and stellar disks. The dark halo-to-disk scale ratio is shorter in the red STGs than in the blue ones, but in a majority of all STGs this ratio is under 2. The optical color $(r-i)$ of the superthin galaxies correlates with their rotation curve maximum, vertical velocity dispersion in stellar disks, and mass of the dark halo. We conclude that there is a threshold central surface density of 50 $M_{\odot} \mathrm{pc}^{-2}$ below which we do not observe very thin, rotationally supported galactic disks.
\end{abstract}

Keywords: galaxies: structure, galaxies: edge-on, galaxies: LSB

\section{INTRODUCTION}

The thinnest disk galaxies have been studied since a long time because of their unusual appearance, e.g., VorontsovVelyaminov (1967); Goad \& Roberts (1979); Karachentsev et al. (1993); Kautsch et al. (2006). These superthin galaxies (STGs) are traditionally defined to have major-to-minor axial ratios larger than nine $(a / b>9)$ when seen edge-on (Goad \& Roberts 1979, 1981). These galaxies are a subgroup of flat disk galaxies and are rare (Karachentsev et al. 1999; Makarov et al. 2001; Mitronova et al. 2005; Kautsch et al. 2006; Bizyaev et al. 2014). Bizyaev et al. (2017) (B17 hereafter) found their fraction is almost 4 per cent among the late-type morphological galaxy class Sd, and 5 per cent among the Sd class in the Kautsch et al. (2006) catalogue. Hence, the STGs represent the 'faint end' of 'flat' and very thin disk galaxies analyzed and reviewed by Karachentsev et al. (1993, 1999); Kautsch (2009).

The main mystery of STGs is their appearance. It is unclear what processes create these thin stellar disks and subsequently what evolution preserves the disks from morphological distortion. Under normal circumstances, galactic stellar disks are under the influence of efficient disk heating processes. These external and internal processes increase the disk thickness and produce the "regular" morphology of the majority of today's disk galaxies (Kormendy 1983;

Corresponding author: Dmitry Bizyaev

dmbiz@apo.nmsu.edu 
Naab \& Burkert 2003; Sotnikova \& Rodionov 2003; Kormendy \& Fisher 2005; D'Onghia et al. 2006; Bournaud et al. 2007; Yoachim \& Dalcanton 2008; Bullock et al. 2008; Kazantzidis et al. 2008, 2009; Purcell et al. 2009; Khoperskov et al. 2010). In this article, we focus on spectroscopic observations of STGs and how those may help explain the resistance of STGs against morphological transformations and disk thickening.

Only a few nearby superthin galaxies have been studied in detail so far. Multi-wavelength data obtained for a 'prototype' STG UGC 7321 (Matthews et al. 1999; Matthews 2000; Matthews \& Wood 2001; Uson \& Matthews 2003) allowed authors to conclude that this galaxy contains a large fraction of dark matter and a low surface brightness (LSB) stellar disk. Kautsch (2009); Bizyaev et al. (2017) came to conclusions that the STGs are LSB galaxies.

It appears that the majority of STGs and simple disk galaxies is located in quiet environments, which may prevent them from external perturbations (Kautsch et al. 2009). B17 noticed that small STGs avoid cosmological filaments, which indicates that these blue, under-evolved galaxies avoid the high density environment. The latter is in agreement with similar conclusions made for LSB galaxies by Rosenbaum \& Bomans (2004); Rosenbaum et al. (2009). Karachentsev et al. (2016) discovered that flat galaxies with very thin disks have fewer satellite companions than regular spiral galaxies. Moreover, spectroscopic studies of small samples of superthin galaxies (Goad \& Roberts 1981; van der Kruit et al. 2001; Matthews \& Uson 2008; O'Brien et al. 2010; Bizyaev et al. 2017; Banerjee \& Bapat 2017; Kurapati et al. 2018) indicate that the galaxies grew up and evolved in rather unperturbed cosmological environments (B17) and hence, allow us to study the galactic disk formation disentangled from the consequent dynamical evolution.

All current considerations about STGs point to the conclusion that dark matter (DM) plays the dominant role in shaping these objects and keeps them stable. Banerjee \& Bapat (2017) noticed that special structural features of dark matter halos in STGs like their compactness, may be responsible for the formation of their thin, LSB disks.

Recent studies found evidence that massive dark halos can press the galactic stellar disks of STGs into their thin shape with low stellar volume density. Zasov et al. (1991, 2002); Kregel \& van der Kruit (2005); Sotnikova \& Rodionov (2006); Khoperskov et al. (2010) found a correlation between the relative disk thickness and the relative mass of a spheroidal DM halo. Other numerical simulations demonstrate that LSB galaxies prefer to form in dark matter halos with long spatial scales (Maccio et al. 2007). Moreover, Di Paolo et al. (2019) studied 72 LSB galaxies and found that the extension of stellar disks (i.e., their disk scale length) increases with larger dark halo scale lengths. Also B17 suggest a rather long dark halo scale lengths for the STGs, in agreement with Maccio et al. (2007). At the same time, Banerjee \& Jog (2013); Banerjee \& Bapat (2017); Kurapati et al. (2018) conclude that dark halos of a few studied STGs have short scale length if expressed in the stellar disk scales.

Banerjee \& Bapat (2017); Kurapati et al. (2018) concluded that the halo compactness seems to have an important role in shaping the stellar disks in STGs, although the sample they worked with was very limited. Simulations by Di Paolo et al. (2019) showed that the rotational velocity of the stellar disks is proportional to the halo-to-disk scale ratio. This means that a stellar disk rotates slow when its DM halo is small and compact, and the stellar disk is thin and extended. Kormendy \& Freeman (2016) noticed that the dark matter halos in late-type spiral and dwarf spheroidal galaxies have smaller core radii and higher central densities, which means their dark halos are compact. In contrast, observations of nearby LSB galaxies indicate that the dark matter halo profiles are cored rather than compact ("cusped") (Kuzio de Naray \& Kaufmann 2011; Salucci et al 2012), suggesting long dark halo scales. Kormendy \& Freeman (2004, 2016); Di Paolo et al. (2019) found that the central surface density of the dark matter halos is constant in a very wide range of objects with various absolute magnitudes ( $M_{B}$ from -5 to $-22 \mathrm{mag}$ ). In this case, the only feature of the dark halos that affects the evolution of the baryonic matter is the scale length of the dark halo (setting aside the asymmetry and interaction between different halos).

The further investigation of possible connections between the properties of the dark halos and the stellar disks should give a valuable feedback to disentangle those discrepancies and feed modern cosmological simulations. Particularly, many STGs are found in unperturbed conditions that did not change significantly over the lifetime and thus provide a well-defined test pad for those simulations. A large number of STGs is required to make reliable, statistically valuable conclusions.

Public surveys provide us with a way to select the STGs, but the spectroscopic data are insufficient to study a large STG sample. Here we rely on a sample of verified edge-on galaxies by Bizyaev et al. (2014) ('Edge-on disk Galaxies 
Table 1. Spectral Observations of Superthin Galaxies. Name, date of observations, total exposure time, and heliocentric radial velocity $\left(\mathrm{km} \mathrm{s}^{-1}\right)$. Note that the names of the galaxies from the EGIS catalogue contain RA and Dec coordinates of their center in decimal degrees.

\begin{tabular}{llll}
\hline Object & Date & $\begin{array}{l}\text { Exposure } \\
\mathrm{min}\end{array}$ & $\begin{array}{l}\mathrm{RV} \\
\mathrm{km} \mathrm{s}^{-1}\end{array}$ \\
\hline EON_105.825_13.464 & 31 Jan 2016 & 40 & 20373 \\
EON_11.160_-11.189 & 29 Aug 2019 & 25 & 8106 \\
EON_115.887_31.535 & 01 Feb 2019 & 15 & 3750 \\
EON_116.146_18.328 & 31 Jan 2016 & 35 & 14604 \\
EON_118.918_28.743 & 01 Feb 2019 & 12 & 6434 \\
... Table is published in its entirety & \\
\multicolumn{4}{c}{ in the electronic edition. ... } \\
\hline
\end{tabular}

In SDSS', hereafter EGIS ${ }^{1}$ ) A part of these galaxies was observed in the near-infrared (NIR) by Bizyaev et al. (2020). In this paper we report results from our long-term spectroscopic campaign, as well as an additional NIR photometry.

The cosmological framework adopted throughout this paper is $H_{0}=72 \mathrm{~km} \mathrm{~s}^{-1} \mathrm{Mpc}^{-1}, \Omega_{m}=0.3$, and $\Omega_{\Lambda}=0.7$.

\section{THE SAMPLE OF SUPERTHIN EDGE-ON GALAXIES}

Our sample of superthin galaxies was selected using the same criteria described in Bizyaev et al. (2017). We used the EGIS (Bizyaev et al. 2014) catalogue to select galaxies with $h / z_{0}>9$ in the $r$-band. We also added several galaxies with visually thin disks with the bluest colors among the EGIS sample, even if their thickness did not meet the $h / z_{0}$ criterion.

We targeted some of selected galaxies for NIR photometric observations (see below) and attempted to observe more objects to enhance the NIR sample in coordination with the sample by Bizyaev et al. (2020).

\subsection{Spectral Observations of Superthin Galaxies}

We continued observations in the same manner as described by Bizyaev et al. (2017). We were able to collect spectra of 138 objects with the Dual Imaging Spectrograph (DIS) on the 3.5m telescope at the Apache Point Observatory (APO) between December 2014 and August 2019. All observations were conducted in the high-resolution mode (B1200/R1200 grating), which provides the spectra resolution of 5000. In addition to the $\mathrm{H} \alpha$ emission, we can see other major emission lines (H $\beta,[\mathrm{OIII}] 4959,5007 \AA$, [NII] $6548,6583 \AA, \&[\mathrm{SII}] 6717,6731 \AA)$ in about three quarter of all galaxies. The deterioration of blue DIS spectra after 2017 because of condensation in the blue DIS dewar made the blue spectra less useful for kinematic studies.

Similar to the observations described by B17, each galaxy was observed with one to three exposures, from 15 to 60 minutes long. A Helium-Neon-Argone wavelength calibration lamp was observed immediately after each galaxy at the same position in the sky. Spectrophotometric standard stars were observed every night. Each observing night was accompanied with a set of biases and dome flats. The data reduction was performed with IRAF standard tools, including bias subtraction, flat fielding, wavelength calibration, sky line and sky background subtraction, cosmic ray removal, and flux calibration. Same as in Bizyaev et al. (2017), the typical accuracy of the radial velocity is $12 \mathrm{~km} \mathrm{~s}^{-1}$.

Table 1 shows the object name (in the EGIS catalogue), date of observations, total exposure time, and our heliocentric radial velocity in $\mathrm{km} \mathrm{s}^{-1}$.

\subsection{Near Infrared Photometry of Superthin Galaxies}

The paper Bizyaev et al. (2020) describes near infrared photometry of 49 galaxies from our list. We conducted additional observations of 18 galaxies with the NICFPS camera on the $3.5 \mathrm{~m}$ telescope at the Apache Point Observatory (APO). Each galaxy was observed in a sequence of 20 to 60 short dithered exposures that prevented the center of galaxies saturate, and gave sufficient signal to combine the images for estimating the sky background. We ensured that the overall exposure time is sufficient for providing with images of the galaxies visually extended similar to corresponding SDSS images. Flat fielding and image co-adding was done using the IRAF standard packages. Similar

\footnotetext{
${ }^{1}$ http://users.apo.nmsu.edu/ dmbiz/EGIS/

https://www.sao.ru/edgeon/catalogs.php?cat=EGIS
} 
Table 2. Objects, Date of observations, Filter, Exposure time and seeing FWHM in arccsec.

\begin{tabular}{lllll}
\hline Object & Date & F & Exp. & FWHM \\
\hline \hline EON_119.500_17.903 & 02 Dec 2015 & H & $40 \times 25$ sec & 1.61 \\
EON_123.495_45.742 & 02 Dec 2015 & H & 40x25 sec & 0.93 \\
EON_128.304_51.316 & 02 Dec 2015 & H & $40 \times 15$ sec & 1.17 \\
EON_129.751_27.821 & 02 Dec 2015 & H & $40 \times 25$ sec & 1.09 \\
EON_132.574_3.497 & 02 Dec 2015 & H & $40 x 30$ sec & 1.20 \\
... Table is published in its entirety & \\
in the electronic edition. ... & \\
\hline
\end{tabular}

to Bizyaev et al. (2020), we applied photometric calibration of the images via non-saturated stars in the fields identified also in the 2MASS survey. Typically we used seven stars in each field and applied the 2MASS-MKO transformation from Legget et al. (2006). The NIR observations are summarized in Table 2.

\section{PROPERTIES OF OUR GALAXIES}

\subsection{Observing Radial Velocities and Rotation Curves}

The rotation curves were obtained from the final, wavelength calibrated and sky subtracted spectra. The emission line profiles in the spectral direction were fitted by a gaussian. The gaussian center position gave us the radial velocity estimate. The uncertainty of the gaussian centroid position combined with the uncertainty of the wavelength calibration in quadratures were used as the uncertainty estimate for individual data points on the rotation curves.

The obtained rotation curve was folded in the spatial direction. The resulting curve was fitted with a third-order polynomial after which 2 to 3 sigma outliers were removed. The spatial position of the center was slightly varied and fitted to make both sides of the curve show the lowest scatter of the radial velocities. The procedure was repeated iteratively until finding the best-fit rotation curve. We also considered to utilize different approximation of the rotation curve shape using equations from Courteau (1997), and came to the same resulting observing points on the rotation curves after removing outliers using the procedure described above. The rotation curve maximum $V_{\max }$ is determined as the highest data point value on the observing rotation curve.

HyperLeda database $^{2}$ (Makarov et al. 2014) contains radial velocity data for many galaxies from our spectral sample. There are 98 optical redshift measurements for 71 our galaxies. 52 of them were reported by SDSS survey (Alam et al. 2015). The mean radial velocity difference (after rejection of some highly deviating points) is $-1.4 \pm 2.9 \mathrm{~km} \mathrm{~s}^{-1}$ with a standard deviation of $27 \mathrm{~km} \mathrm{~s}^{-1}$, which indicates an excellent agreement. In addition, HI-observations are available for 52 galaxies from our sample. The data are taken from the HI-catalogue of the Extragalactic Distance Database EDD by Courtois et al. (2009) (40 galaxies), from the ALFALFA survey by Haynes et al. (2018) (31 objects) and from Springob et al. (2005) (24 galaxies). HI-observations of edge-on spirals by Giovanelli et al. (1997) were performed for 14 galaxies and HI-survey of highly flattened, edge-on, pure disk galaxies by Matthews \& van Driel (2000) gave us data for comparison for 13 objects. After rejecting a few outliers the mean difference between HI-data and the systematic velocity from the rotation curve is $7.9 \pm 2.3 \mathrm{~km} \mathrm{~s}^{-1}$ with a standard deviation of $29 \mathrm{~km} \mathrm{~s}^{-1}$. Having in mind methodological differences of systemic velocity determination in HI and long-slit observations, we conclude that our data are in a good agreement with radio observations.

Thirty-nine galaxies from the spectral list do not have radial velocities in the NED database ${ }^{3}$. Sixteen more those galaxies have NED radial velocities different by more than $100 \mathrm{~km} \mathrm{~s}^{-1}$ from those in Table 1 . Thus, we report 40 per cent of new, correct radial velocities for our sample of superthin galaxies. The mean radial velocity difference between the NED and Table 1 for the other 84 galaxies is $-1.4 \mathrm{~km} \mathrm{~s}^{-1}$ with the sigma of $39 \mathrm{~km} \mathrm{~s}^{-1}$. The median difference and the MAD scatter are even lower: -0.4 and $31 \mathrm{~km} \mathrm{~s}^{-1}$, respectively.

Besides the redshifts, the HyperLeda database provides information about kinematics of galaxies as HI-line-width measurements and the rotation curves maximum. Fig. 1 shows comparison of our $V_{\max }$ measurements with published data on the HI-line-width by its 50 per cent level $W_{50}$ : 24 galaxies from Springob et al. (2005) are designated with green circles, 42 blue squares correspond to the HI data by Courtois et al. (2009), 31 cyan diamonds show the data

\footnotetext{
2 http://leda.univ-lyon1.fr

${ }^{3}$ https://ned.ipac.caltech.edu
} 


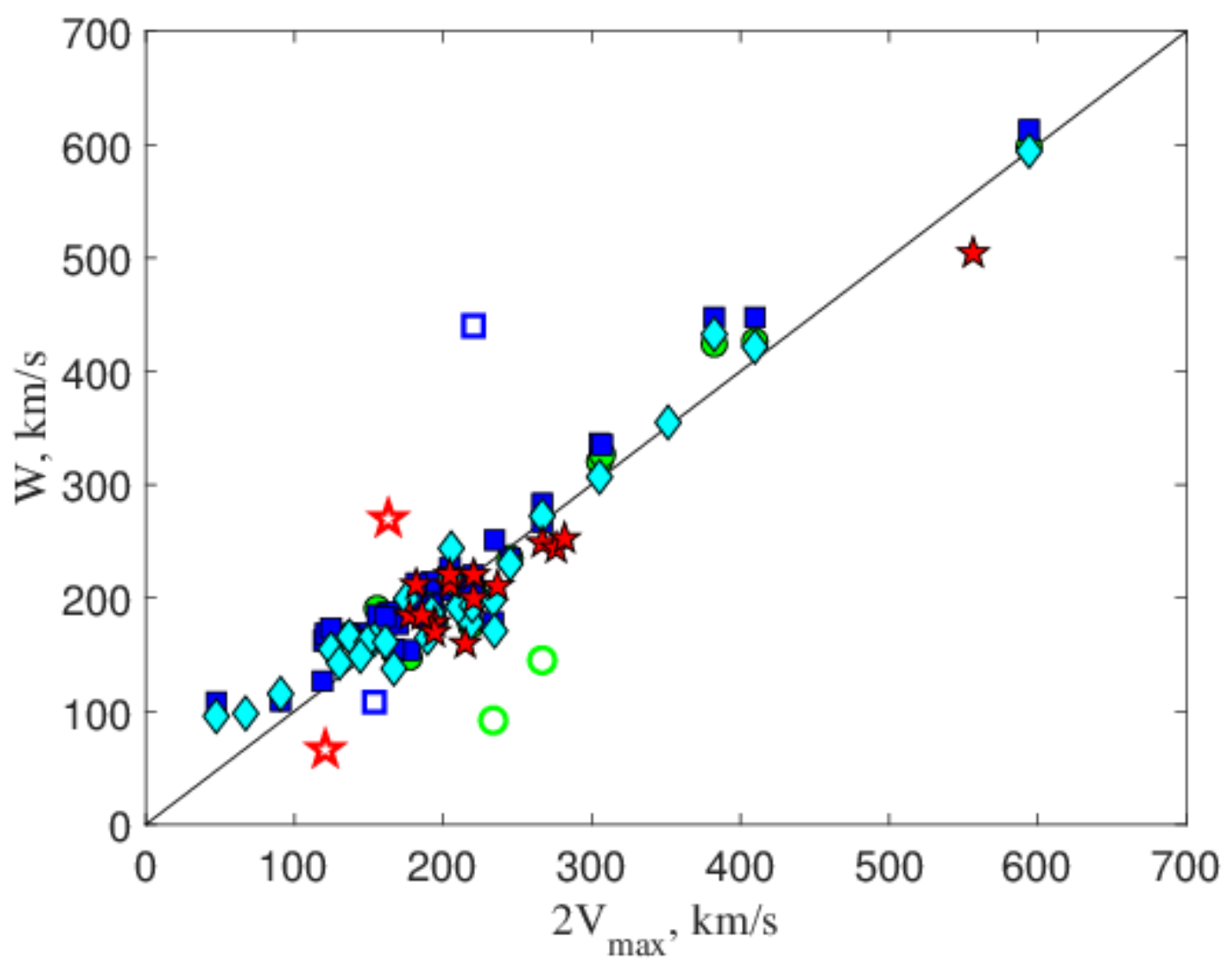

Figure 1. Comparison of our $V_{\max }$ with published widths of radiolines by their 50 per cent level $W_{50}$. The green circles designate the objects from Springob et al. (2005), the blue squares correspond to Courtois et al. (2009), the cyan diamonds show sample from Haynes et al. (2018), and the red stars represent direct rotational velocity maxima from published rotation curves (see text). The data points rejected from the analysis are designated by open symbols. The solid black line corresponds to the relation $W_{50}=2 V_{\max }$.

Table 3. Comparison source, number of common galaxies $\mathrm{N}$, linear regression parameters $a$ and $b$ for $V_{\max }=a+b\left(W_{50} / 2\right)$, and the regression residuals $\sigma_{V}$. Designations of the sources follows: S05 - Springob et al. (2005), EDD - Courtois et al. (2009) and ALFALFA - Haynes et al. (2018). The RC refers to the individual data sources mentioned in the paper, see text.

\begin{tabular}{lcccc}
\hline Source & $\mathrm{N}$ & $\begin{array}{c}\mathrm{a} \\
\mathrm{km} \mathrm{s}^{-1}\end{array}$ & $\begin{array}{c}\mathrm{b} \\
\mathrm{km} \mathrm{s}^{-1}\end{array}$ & $\begin{array}{c}\sigma_{V} \\
\mathrm{~km} \mathrm{~s}^{-1}\end{array}$ \\
\hline S05 & 22 & $2.6 \pm 5.7$ & $0.95 \pm 0.04$ & 11.1 \\
EDD & 43 & $0.8 \pm 4.7$ & $0.94 \pm 0.04$ & 11.9 \\
ALFALFA & 31 & $0.5 \pm 5.3$ & $0.98 \pm 0.04$ & 13.3 \\
RC & 15 & $-5.1 \pm 9.0$ & $1.11 \pm 0.08$ & 11.5 \\
\hline
\end{tabular}

from ALFALFA survey (Haynes et al. 2018), and 17 red stars for 14 different galaxies represent direct rotational velocity maximum measurements from rotation curves. The rejected data points are designated by open symbols. The black line marks the $W_{50}=2 V_{\max }$ correspondence. Table 3 summarises results of a linear regression between our measurements of $V_{\max }$ and literature data on $W_{50}$ in the form of $V_{\max }=a+b\left(W_{50} / 2\right)$. The zero-point $a$ is essentially zero, while the slope $b$ is equal to one, statistically.

Data on the rotation curves maximum for a few objects show contradictions between different literature sources and our observations. We comment on them with possible explanation below.

EON_019.768_-00.139 (PGC 4734, RFGC 296, UGC 847). The spectrum of this object by Springob et al. (2005) has low signal-to-noise ratio that led to significant underestimation of its $W_{50}=145 \mathrm{~km} \mathrm{~s}^{-1}$. 
EON_225.915_42.126 (PGC 53744, RFGC 2908, UGC 9681). This galaxy forms a close pair with PGC $53758=$ UGC 9684. The angular separation is 2.1 arcmin only. As a result, all HI-data are contaminated by the companion.

EON_032.766_06.667 (PGC 8353, RFGC 469, UGC 1677). Most of the radio data for this galaxy are wrongly associated with HI-emission from a nearby galaxy UGC 1670 with $V_{h} \sim 1600 \mathrm{~km} \mathrm{~s}^{-1}$. However, ALFALFA survey (Haynes et al. 2018) reports reliable HI-velocity and line-width estimation for our object.

\subsection{Structural Parameters}

In this paper we make use of the structural parameters of galactic disks estimated both in the optical and NIR bands using SDSS archival photometry processed by Bizyaev et al. (2017) and NIR photometry from Bizyaev et al. (2020) and this paper. The optical and NIR disk scales for the galaxies were estimated with pipelines described by Bizyaev et al. (2014) and Bizyaev et al. (2020). The disk scales were estimated taking the spatial resolution of optical and NIR photometric observations into account. We ensure that the limited spatial resolution of the imaging does not affect the estimated vertical scale heights in out galaxies systematically, according to the conclusions made by Bizyaev et al. (2014). In addition to the scales reported in the published papers, we estimate the vertical and radial disk scales from the NIR photometry of 18 galaxies reported in this paper. The structural parameters are shown in Table 4.

Despite the performed NIR observations for some of galaxies from the spectroscopic sample, most of them do not have the disk scales in the NIR. Fortunately, Bizyaev et al. (2020) showed that the optical and NIR scales are connected, and we can estimate the NIR scales using SDSS data from Bizyaev et al. (2014) using the following relations:

$$
\begin{aligned}
h(H) & =0.481( \pm 0.099) h(r), \\
z_{0}(H) & =0.855( \pm 0.138) z_{0}(r)
\end{aligned}
$$

As it was noticed by Bizyaev et al. (2020), the disk scale height is almost the same in the NIR and optical measurements, while the near-infrared disk scale length is significantly shorter. The latter can not be explained by the dust attenuation effects alone, and reveals a real difference in distribution of young and old stellar populations though the galactic disks.

The central surface brightness is affected by the dust extinction and we were not surprised to see a large scatter for the relation between the optical $\mu_{r}$ and NIR $\mu_{H, K}$ central surface brightness. Incorporating the optical color $(r-i)_{0}$ helps reduce the uncertainties of such a cross-calibration. We perform a two-dimensional fitting to the data from Bizyaev et al. (2020) and find the following relation:

$$
\mu_{H}=12.227-7.043(r-i)_{0}+0.415 \mu_{r},
$$

where the $(r-i)_{0}$ is the SDSS color of galaxies corrected for the Milky Way extinction from Bizyaev et al. (2014). Although the relation provides a rather coarse cross-calibration with the uncertainty of $0.7 \mathrm{mag}$, it is helpful for including the galaxies without NIR observations into the further analysis, given we do not use photometric data to convert the surface brightness to surface density directly.

The rotation curve maximum, optical and NIR structural parameters are shown in Table 4. The 'NIR flag' Fnir designates the source of the NIR protometry for the galaxy, and can be ' $h$ ' or ' $\mathrm{k}$ ' when the APO 3.5m data are used, ' $\mathrm{H}$ ' or ' $\mathrm{K}$ ' when the data are taken from Bizyaev et al. (2020), or ' $\mathrm{F}$ ' if equation (1) was applied. The distances in Table 4 correspond to our radial velocities corrected for the CMB motion according to the $\mathrm{NED}^{4}$.

Fig. 2 demonstrates the rotation curves of all galaxies from Table 1. The red solid curve shows the Milky Way galaxy (Sofue et al. 1999). The lower panel demonstrates our rotation curves with the radius normalized by the disk scale length in the $r$ band. Fig. 2 reveals that the majority of our superthin galaxies have rotation curves with shallow central parts, which is an indication of the dominance of DM in STGs. The rotation curve maximum, if detected, is reached at several times farther from the center than in the regular galaxies, which is a typical feature of low surface brightness galaxies.

\subsection{Properties of Rotation Curves in Superthin Galaxies}

Fig. 3 shows the relation between the disk scale length (estimated in the r-bang here) and the maximum rotation velocity $V_{\max }$. We confirm the trend reported by B17. The robust linear fitting to Fig. 3 gives $h \propto V_{\max }^{1.6}$. This result

${ }^{4}$ https://ned.ipac.caltech.edu 

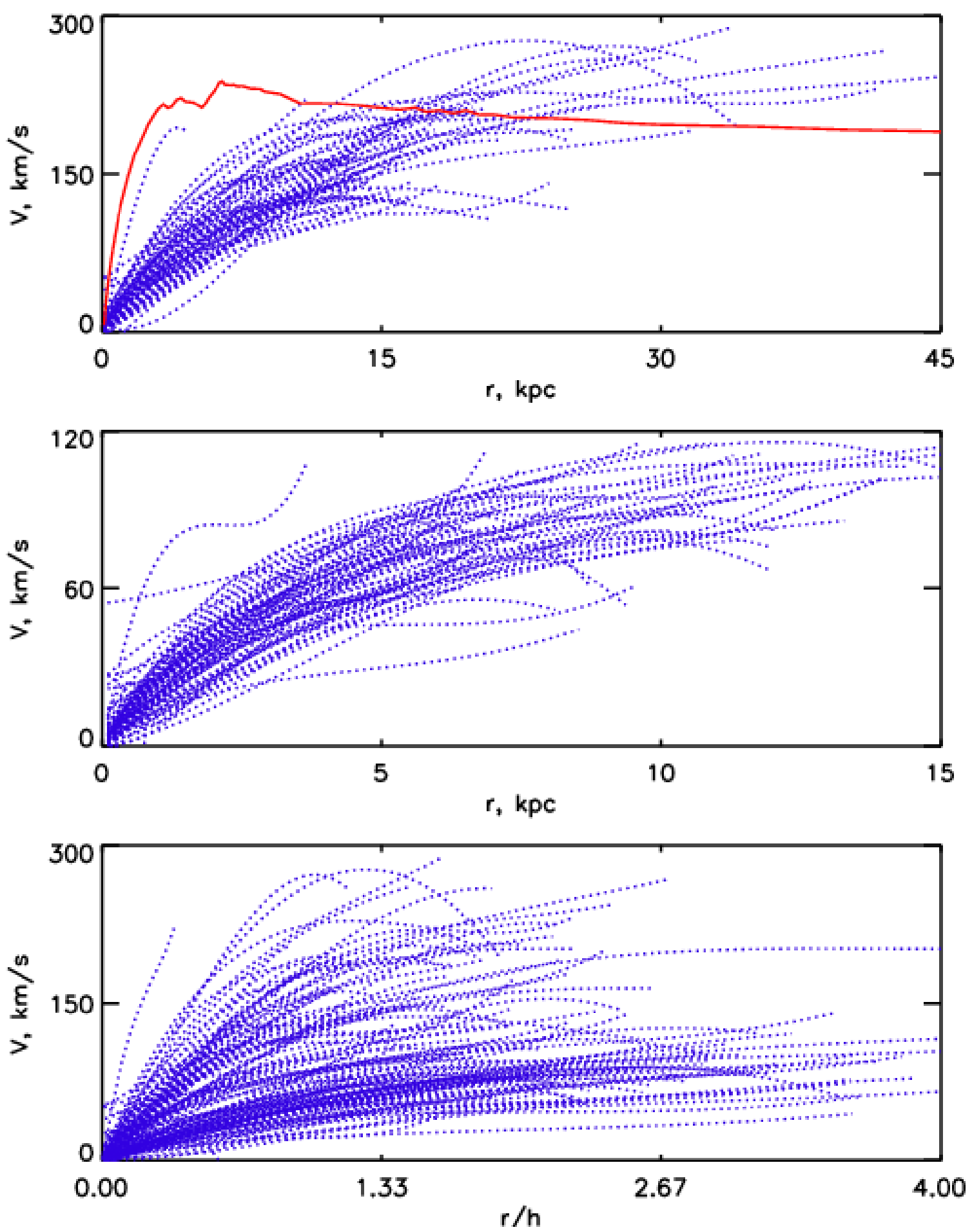

Figure 2. Rotation curves of our 138 superthin galaxies are shown with the blue dotted curves for objects with $V_{\text {max }}>120$ $\mathrm{km} \mathrm{s}^{-1}$ (upper panel) and $V_{\max } \leq 120 \mathrm{~km} \mathrm{~s}^{-1}$ (middle panel). The solid red curve demonstrates the Milky Way's rotation curve. The lower panel repeats the plots above for the same rotation curves with radius normalized by the $r$-band disk scale length. 
Table 4. Parameters of Superthin Galaxies. The columns show the name of galaxies, distance in Mpc that corresponds to the radial velocity corrected for the CMB motion, the radial scale $h$ and its uncertainty from the EGIS catalogue, in kpc, the vertical scale $z_{0}$ and its uncertainty from the EGIS catalogue, in $\mathrm{kpc}$, the same scales and their uncertainties from NIR opbservations (see text), the $(r-i)_{0}$ color, the maximum of the rotation curve velocity, and the "Fnir" flag (see text).

\begin{tabular}{lllllllllllll}
\hline Name & D,Mpc & hopt & dhopt & z0opt & dz0opt & hnir & dhnir & z0nir & dz0nir & $(\mathrm{r}-\mathrm{i})$ & $V_{\max }$ & Fnir \\
\hline EON_105.825_13.464 & 285.14 & 7.881 & 1.297 & 0.789 & 0.220 & 3.791 & 0.623 & 0.675 & 0.188 & 0.379 & 142 & $\mathrm{~F}$ \\
EON_11.160_-11.189 & 108.10 & 4.368 & 0.069 & 0.889 & 0.266 & 2.101 & 0.034 & 0.760 & 0.227 & 0.232 & 131 & $\mathrm{~F}$ \\
EON_115.887_31.535 & 54.43 & 2.892 & 0.018 & 0.520 & 0.076 & 1.391 & 0.009 & 0.445 & 0.065 & 0.149 & 86 & $\mathrm{~F}$ \\
EON_116.146_18.328 & 205.64 & 4.833 & 0.127 & 0.804 & 0.081 & 2.325 & 0.061 & 0.687 & 0.069 & 0.405 & 154 & $\mathrm{~F}$ \\
EON_118.918_28.743 & 92.00 & 2.643 & 0.222 & 0.852 & 0.143 & 1.271 & 0.107 & 0.729 & 0.122 & 0.156 & 62 & $\mathrm{~F}$
\end{tabular}

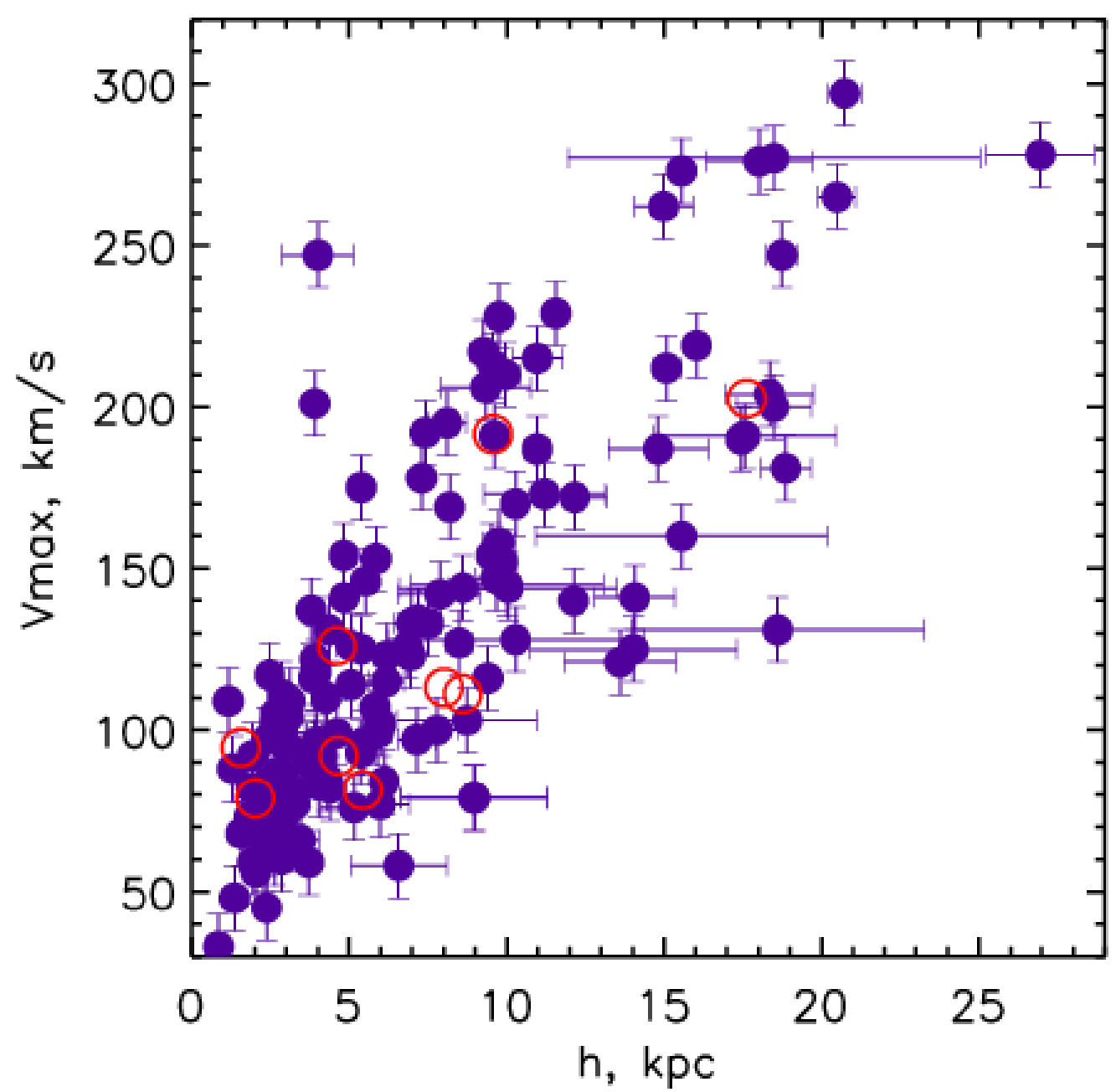

Figure 3. The rotation curve maximum $V_{\max }$ versus the $r$-band scale length for our (blue bullets) and published (red circles) data.

is in a qualitative agreement with similar relations found by Courteau et al. (2007); Hall et al. (2012) made for large samples of regular disk galaxies. 


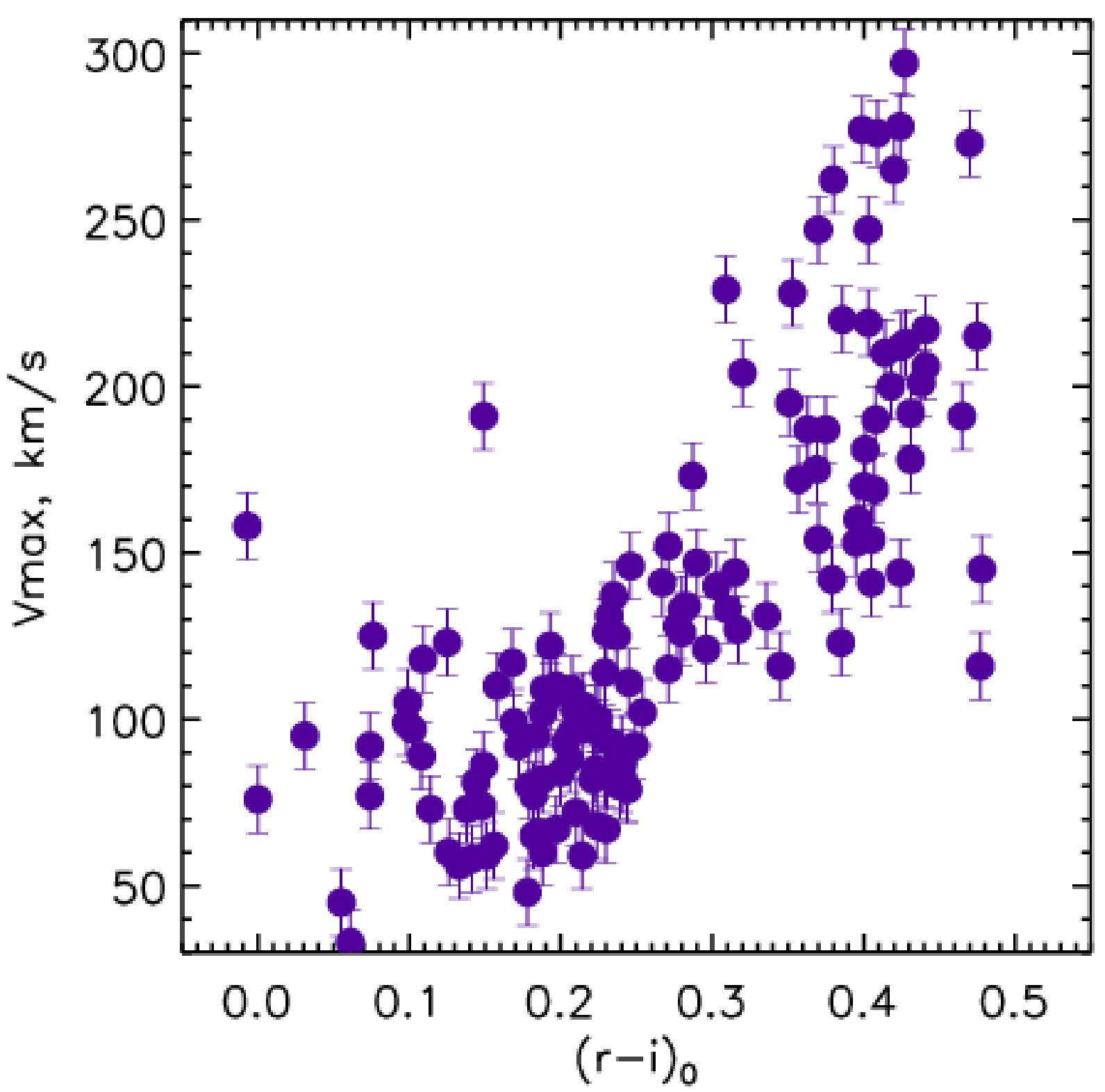

Figure 4. The rotation curve maximum $V_{\max }$ versus the galaxy color $(r-i)_{0}$ corrected for the galactic foreground extinction.

According to B17, the STG is a non-uniform group of galaxies that demonstrates different properties between the red and blue objects: the red galaxies are large and dynamically evolved, while the blue galaxies are small and dynamically cold. Fig. 4 shows that the maximum rotation velocity $V_{\max }$ depends on galactic color, which is in agreement with B17. The blue galaxies with $(r-i)_{0} \leq 0.24 \mathrm{mag}$ are mostly low massive, with low $V_{\max }$ independent of color. The red galaxies with $(r-i)_{0}>0.24 \mathrm{mag}$ are large, and we can see a positive correlation between their $V_{\max }$ and color. Note that the $(r-i)_{0}=0.24$ mag threshold splits our sample of galaxies in equal halves.

We show examples of blue and red galaxies in Fig. 5. The upper panel demonstrates a slowly rotating blue STG EON_32.766_6.667, while the lower panel shows a large, red, massive STG EON_149.150_20.646. 

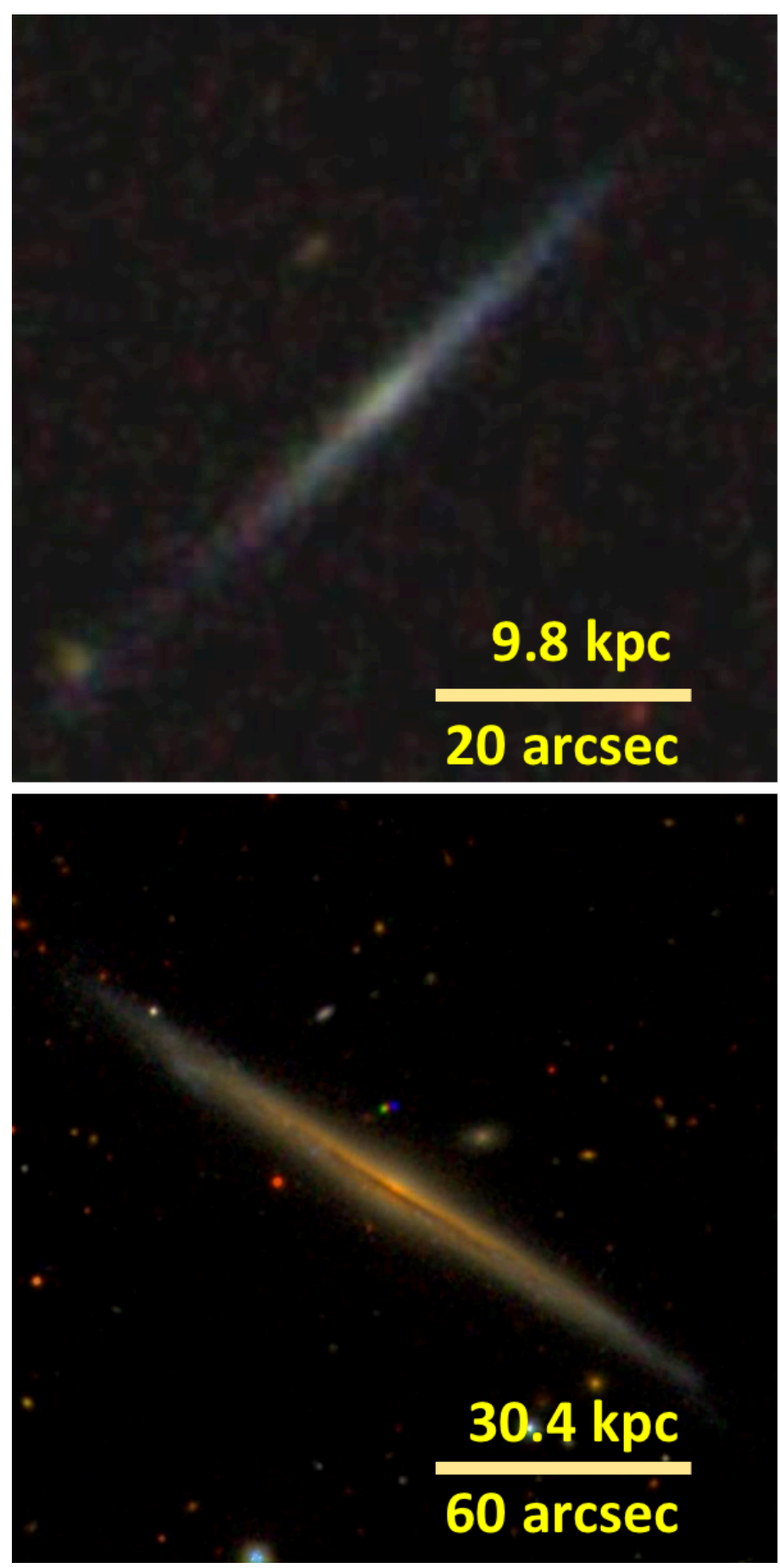

Figure 5. Examples of blue (EON_32.766_6.667, top) and red (EON_149.150_20.646, bottom) superthin galaxies from our sample. The horizontal bar in each panel demonstrates the scale in arcseconds and kpc at the distance of the galaxies.

Significant fraction of additional radial velocity measurements allows us to compare the physical scale length of regular and superthin galaxies, similar to what has been done by B17. We confirm the conclusion by B17 that the STGs have bluer colors for the same scale length as regular galaxies.

\subsection{Stability of Disks and their Thickness}

As it was noticed by B17, a simple galaxy model with an exponential stellar disk and a spherical gravitational potential of halo enables us to estimate the relative disk thickness (Zasov et al. 1991; Kregel et al. 2005; Sotnikova 
\& Rodionov 2006; Bizyaev \& Mitronova 2009; Khoperskov et al. 2010). In B17 we assumed that the disk mass is $M_{d}=2 \pi \Sigma_{0} h^{2}$, and the total mass of the galaxy within four disk scale lengths is $M_{t}=4 V_{\max }^{2} h / G, \Sigma_{0}$ is the central surface density of the disk, $V_{\max }$ is the circular velocity, and $G$ is the gravitational constant. For the disks in equilibrium in the vertical direction, their vertical scaleheight $z_{0}$ is (Spitzer 1942): $\sigma_{z}^{2}=\pi G \Sigma(R) z_{0}$, where $\sigma_{z}$ is the vertical stellar or gas velocity dispersion.

When combined with an assumption of the stellar disk marginal stability (Toomre 1964), the radial stellar velocity dispersion $\sigma_{R}$ is $\sigma_{R}=3.36 G \Sigma(R) / \kappa$, where $\kappa$ is the epicyclic frequency. If we take into account the non-axisymmetric perturbations and finite layer thickness, the minimum radial dispersion should be greater than $\sigma_{R} \geq Q 3.36 G \Sigma(R) / \kappa$, where $Q>1$ is the Toomre parameter The value of $Q \approx 1.4$ is consistent with the empirical criterion of gravitational stability (Kennicutt 1989). The epicyclic frequency at the region of the flat rotation curve is $\kappa=\sqrt{2} V_{\max } / R$, and we get $z_{0} \sim\left(M_{d} / M_{t}\right) h$. At $R=2 h$ the total-to-disk mass ratio is $M_{t} / M_{d} \gtrsim 1.2\left(\sigma_{z} / \sigma_{R}\right)^{2}\left(h / z_{0}\right)(Q / 1.4)^{2}$.

Note that we explore the case with thin disks and low vertical velocity dispersion, and refer to epochs when the galactic disks were mostly gaseous, for which case $\sigma_{R}=\pi G Q \Sigma(R) / \kappa$ (Safronov 1960) and $\left(\sigma_{z} / \sigma_{R}\right)=1$. As it was further noticed by B17, the proximity of the vertical velocity dispersion in the stellar and gas disks, as well as the small disk thickness, can make the pure gas disk stability criterion equation applicable in the case of the superthin disks. In this case $M_{t} / M_{d} \gtrsim 1.1\left(h / z_{0}\right)(Q / 1.4)^{2}$. We assume that the vertical velocity dispersion never falls below $\sigma_{z}=10 / \sqrt{3} \mathrm{~km} / \mathrm{s}$. For $\mu=M_{t} / M_{d}$ and $Q=1.4$ B17 obtain

$$
h / z_{0} \lesssim(81 / \mu) V_{100}^{2}
$$

where where $V_{100}=V_{\max } / 100 \mathrm{~km} \mathrm{~s}^{-1}$.

The relationship in eq.(3) corresponds to a marginally low vertical velocity dispersion in the stellar component of the order of $6 \mathrm{~km} \mathrm{~s}^{-1}$, which is the same as in the star-forming gas component (Kennicutt 1989; Mogotsi et al 2015).

B17 noticed that the eq.(3) does not limit the scale ratio $h / z_{0}$, and we could observe arbitrary thin galaxies under reasonable assumptions on the $\mu$. The lack of observations of extremely thin galaxies could be explained by an additional constraint on the minimum stellar surface density $\Sigma_{*}$ that allows disks to form. In this case the $V_{\max }-$ disk scale ratio relation looks like

$$
h / z_{0}=107 V_{100}^{0.5} / \Sigma_{0, c}
$$

(see B17). The limited sample of galaxies considered by B17 suggests the minimum disk central surface density of 88 $M_{\odot} \mathrm{pc}^{-2}$. Now we have collected a much larger sample of galaxies and can review this figure. Fig. 6 shows the $h / z_{0}$ - $V_{\max }$ diagram for our galaxies. The red dotted line corresponds to the former value of $\Sigma_{0, c}=88 M_{\odot} \mathrm{pc}^{-2}$.

Fig. 6 demonstrates a lower threshold on the limiting central surface density $\Sigma_{0, c}$. It needs to be at most $49 M_{\odot} \mathrm{pc}^{-2}$ to explain the most rightward points on the diagram. At the same time, most of the points meet the threshold set by eq.(4). The outlier below the blue dashed line can be explained by a lower than assumed spherical-to-disk mass ratio $\mu$. We purposely show the galaxies with the $h / z_{0}$ scale ratio below 9 to indicate the trends created by the galaxies on the border of the superthin criterion. Those galaxies were selected among the most blue and visually thin galaxies, without the formal, numerical selection by their thickness.

\section{SIMULTANEOUS ROTATION CURVE AND DISK THICKNESS MODELING}

\subsection{Assumptions for the Modeling}

The rotation curve modeling demonstrates a large variability in the obtained parameters of stellar disks and dark halos between the "maximum disk" and "maximum halo" cases (e.g. van Albada \& Sancisi 1986). Introduction of an information about the vertical velocity dispersion helps constrain the modeling significantly (Angus et al. 2015). In the case of edge-on galaxies, using the disk thickness allows us to constrain the rotation curve modeling (e.g. Khoperskov et al. 2010). Note that in a bulgeless galaxy with one exponential stellar disk with known scale length and a spherical dark matter halo only, the additional constraint from the stellar disk thickness leads to only two free parameters in the modeling: the scale length of the halo and the central surface brightness of the disk. The latter also can be constrained by known central surface brightness and an estimate of the mass-to-light ratio from the galactic color. The dust extinction makes such an estimate very uncertain in edge-on galaxies, and the stellar surface densities estimated from an edge-on galaxy decomposition should be used in practice only as a lower limit. As we mention in §3.1, our galaxies have significantly different scale lengths in their old and young stellar populations traced by the optical and NIR photometry, which can be interpreted as a presence of at least two stellar disks with different scale lengths but similar scale heights. This implies complications that we intend to introduce to the simple single disk-in-halo model. 


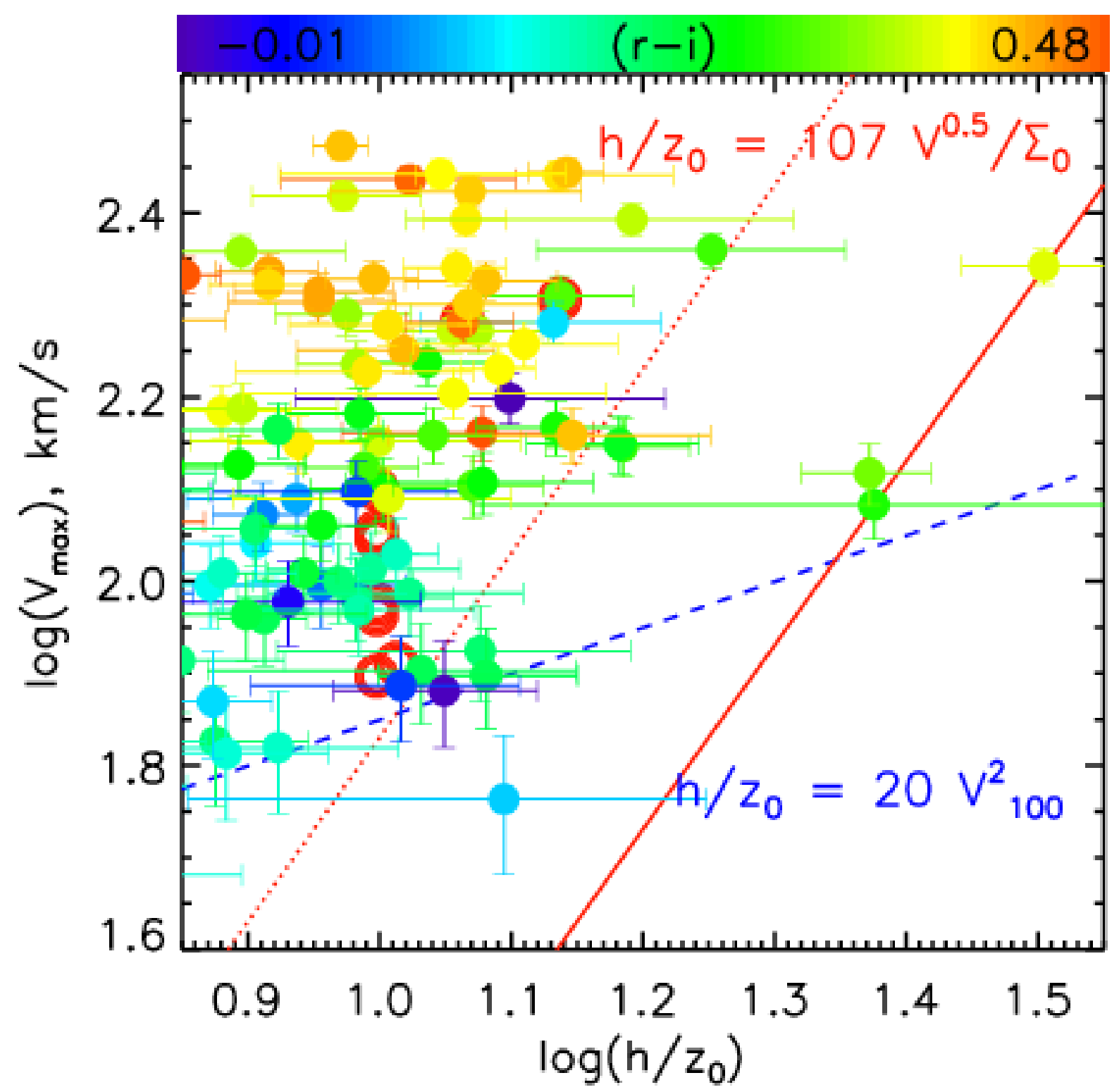

Figure 6. The rotation curve maximum $V_{\max }$ versus the scale ratio $h / z_{0}$. The colored bullets show our sample of galaxies. The symbol color indicates the galactic ( $\mathrm{r}-\mathrm{i})_{0}$ (upper bar). The red open circles designate the data available from literature. The dotted red line marks the case of the disk surface density threshold found by B17. The solid red line indicates our new density threshold of $49 M_{\odot}$ found for our sample. The dashed blue line is the case of the extremely small disk thickness regulated by eq.(3).

Similar to $\S 3.4$ and B17, we assume that the volume density distribution $\rho_{d}$ in the stellar disk follows exponential law along the radius $r$ and isothermal along the vertical direction $z$ :

$$
\rho_{d}(r, z)=\rho_{d, 0} \exp (-r / h) \operatorname{sech}^{2}\left(z / z_{0}\right) .
$$

The dark matter halo is assumed to be spherical and pseudo-isothermal with its density

$$
\rho_{\text {halo }}(R)=\rho_{\text {halo }, 0}\left[1+\left(R / a_{\text {halo }}\right)^{2}\right]^{-1},
$$

where the $\rho_{\text {halo }, 0}$ is the central density, $a_{\text {halo }}$ is the dark matter distribution scale length, and $\mathrm{R}$ is the three-dimensional distance to the center $R^{2}=r^{2}+z^{2}$. In this paper we include the stellar disk thickness into the analysis via the relation 
between the inverse relative disk thickness $h / z_{0}$ and the spherical-to-disk mass ratio shown by Khoperskov et al. (2010). The latter can be parameterized as

$$
M_{s p h} / M_{\text {disk }}=-1.256+0.399\left(h / z_{0}\right) .
$$

The gravitational potential of an exponential disk contributes to the rotation curve as

$$
V_{d}^{2}(R)=4 \pi G \Sigma_{0} h y^{2}\left[I_{0}(y) K_{0}(y)-I_{1}(y) K_{1}(y)\right],
$$

where $\mathrm{y}=0.5 \mathrm{R} / \mathrm{h}, \Sigma_{0}$ is the disk central surface density, $I_{n}(y)$ and $K_{n}(y)$ are the modified Bessell functions of the first and second kind (Binney \& Tremaine 1987; Jimenez et al. 2003).

The contribution of the pseudo-isothermal spherical dark halo is assumed to be

$$
V_{\text {halo }}^{2}(R)=4 \pi G \rho_{0} a_{\text {halo }}^{2}\left[1-\left(\left(a_{\text {halo }} / R\right) \arctan \left(R / a_{\text {halo }}\right)\right)\right]
$$

We anticipate to find an exponential pseudo-bulge in the thin, star forming galaxies, so in rare cases when Bizyaev et al. (2014) suggested a bulge in our galaxy, we described its contribution $V_{b}^{2}$ similar to equations $(5,8)$ with its own bulge scale length $R_{b}$.

The gas contribution to the overall gravitational potential is small in large galaxies like the Milky Way. In small, LSB galaxies its fraction can be noticeable (van der Kruit et al. 2001), and we cannot ignore it in the case of our superthin sample. However, direct measurements of the neutral gas distribution across the disks were performed for just a few, nearby galaxies (Uson \& Matthews 2003; Matthews \& Uson 2008; O'Brien et al. 2010). Fortunately, our galaxies are either large and dynamically evolved, or small and under-evolved (see B17). In the former case we can assume that the galactic gas contribution to the overall gravitational potential is very small. In the latter case we expect to see significant gas contribution, but the gas galactic subsystem resembles the young stellar population by the structural parameters. In this case we can refer to the young stellar disk as to a mix of gas and stars, with the volume density parameterized by equation (5) and the rotation curve contribution described by equation (8).

The resulting rotation curve $V_{\text {all }}$ is the sum of $V_{d}, V_{\text {halo }}$ and $V_{b}$ (if detected) in quadrature $V_{\text {all }}^{2}=V_{d}^{2}+V_{\text {halo }}^{2}+V_{b}^{2}$. We add the asymmetric drift correction Binney \& Tremaine (e.g. in 1987) obtained for the case of gas to the velocity above in quadrature.

Since all our galaxies are seen edge-on, we take into account the projection effects by integrating the $V_{\text {all }}$ along the line of sight without accounting for a small deviation from the perfect edge-on inclination. In this case we assume that the observable model velocity $V_{\bmod }$ is

$$
V_{\text {mod }}(x)=\int_{l o s} V_{\text {all }}(r) \rho_{g}(l) d l / \int_{l o s} \rho_{g}(l) d l
$$

Here $x$ is the distance from the center along the major axis, $\rho_{g}$ is the emitting gas density, which we assume to be distributed similar to $\rho_{d}$, and $l$ is the emitting element depth along the line of sight, $l^{2}=r^{2}-x^{2}$. The depth $l$ is normalized such that $l=0$ at the near edge of the disk.

Matthews \& Wood (2001); MacLachlan et al. (2011) came to a conclusion that small and thin LSB galaxies have low overall dust extinction. Their face-on optical depth is much less than unity. The dust scale height in those galaxies is comparable to their stellar scale height. We do not neglect the dust extinction, given its contribution in regular galaxies can significantly change the shape of rotation curve in the inner disk regions (Bosma et al. 1992; Zasov \& Khoperskov 2003). We assume that the dust forms an exponential disk and that the dust extinction coefficient (Xilouris et al. 1999) can be described as

$$
\kappa(r, z)=\kappa_{0} \exp \left(-r / h_{\text {dust }}\right) \operatorname{sech}^{2}\left(z / z_{\text {dust }}\right),
$$

where $\kappa_{0}$ is the central value. The resulting dust extinction $\tau=\int_{l o s} \kappa d l$. We expect that the $h_{d u s t}$ can vary between $h$ and $2 h$, and the $z_{d u s t}$ vary between $0.5 z_{0}$ and $z_{0}$ (Xilouris et al. 1999; Matthews et al. 1999; Yoachim \& Dalcanton 2006; Bianchi 2007). We note in advance that the resulting extinction is found to be small, and we cannot notice significant difference between the cases of $h$ and $2 h$ for the dust scale length and between $0.5 z_{0}$ and $z_{0}$ for the dust scale height in the modeling described below in the paper. Also, the reasonable range of the dust extinction values in equation (11) that brings up the $\tau_{V}$ at the center of the disk integrated along the vertical direction (i.e. its face-on value) in the $V$-band between 0 and 1 does not affect the resulting modeled rotation curves significantly. For certainty, 
we assume that $h_{\text {dust }}=h$ and $z_{\text {dust }}=z_{0}$ and the central face-on $\tau_{V}=0.5$ in the models described below, unless the dust extinction parameters were allowed to vary as free parameters (Models 6 and 7 ). We neglect the light scattering because it makes only a small effect in the detailed radial transfer simulations on edge-on galaxies (Bianchi 2007). The dust extinction $\tau$ is added to equation (10). The finite FWHM for the spectral observations is taken into account. The model rotation curve is convolved with a Gaussian kernel that corresponds to the reported seeing (see Table 2), in order to obtain the final rotation curve before the comparison with the observable one.

\subsection{The Model Constraints and Free Parameters}

The set of equations (5-11) allows us to build model rotation curves $V_{\text {mod }}$ and compare them to our observations $V_{\text {obs }}$. We employ additional constraints for the vertical structure information that helps mitigate ambiguities in the rotation curve modeling. We develop several models with different sets of parameters and constraints from observations. The variety of models helps us understand how different assumptions affect the model output. The model parameters were evaluated via the chi-square optimization. We minimized the sum $\Sigma\left(V_{\text {mod }}-V_{o b s}\right)^{2} / \sigma_{o b s}$, where the $\sigma_{o b s}$ designates uncertainties of the rotation curve points.

We incorporate the optical and NIR structural parameters from Table 4. We indirectly use the stellar densities estimated from the optical (Bizyaev et al. 2014) and NIR (Bizyaev et al. 2020) central surface densities via the massto-light values tabulated by Bell et al. (2003). The estimated densities set the lower limits, while the real density values should be higher due to the extinction corrections and the gas contribution.

\section{Model 0}

This is an over-simplified, toy model that assumes only one disk whose scale length and height correspond to the optical observations. It has two free parameters (in the case of no bulge): the dark halo scale length $a_{\text {halo }}$ and the disk central surface density. While the model does not take into account additional constraints that come from the optical or NIR observations, it helps establish fiducial chi-square values for each galaxy, which can be used for comparison with the other models.

\section{Model 1}

Here we assume the presence of two disks - young and old. The young disk has the $h$ and $z_{0}$ estimated from optical SDSS images, while the old one has the scales estimated from the NIR data. We have two surface density parameters instead of one, so the number of free model parameters increases by one with respect to the Model 0.

Model 2

This model has the same parameters as the Model 1, but the halo-to-disk mass ratio does not follow the eq.(7). Instead, this is a free parameter of the model.

\section{Model 3}

In this case we assume a single stellar disk, but its scale length is unknown and is a free parameter. The unknown scale length $h_{d}$ varies between the 'young' $h$ and 'old' $h_{n i r}$ values. The single disk surface density is also a free parameter. The assumptions can be interpreted as the presence of an infinite number of disks, whose 'effective' scale length $h_{d}$ and total central surface brightness are evaluated in the model.

Model 4

This model is the same as Model 2, but the central surface densities of the old and young stellar disks have additional constraints. They are limited by the minimum surface densities estimated from the optical and NIR photometry. The upper limits are 2 magnitudes higher than the lower limits, which accounts for a possible dust extinction and for the gas addition.

\section{Model 5}

Here we modified the Model 3 by adding constraints on the central surface density of the disk the same way as for the Model 4, where the minimum surface densities were estimated from the photometry.

Model 6

We modified Model 5 by adding the central dust absorption $\kappa_{0}$ from equation (11) as a free parameter, while all other parameters and constraints were kept unchanged.

\section{Model 7}

Here we added the central dust absorption $\kappa_{0}$ as a free parameter to the Model 0.

The parameters of the models are summarized in Table 5. Note that we used one additional free parameter - the bulge scale - for the galaxies with bulges. The mass of the bulge was estimated by assuming the bulge-to-disk mass ratio follows the bulge-to-disk luminosity ratio from the EGIS catalogue. 
Table 5. Parameters of the models

\begin{tabular}{llr}
\hline Model & Free parameters & Constraints \\
\hline Model 0 & $\Sigma_{0}, a_{\text {halo }}$ & None \\
Model 1 & $\Sigma_{0, \text { young }}, \Sigma_{0, \text { old }}, a_{\text {halo }}$ & None \\
Model 2 & $\Sigma_{0, \text { young }}, \Sigma_{0, \text { old }}, a_{\text {halo }}, M_{\text {halo }} / M_{d}$ & None \\
Model 3 & $\Sigma_{0}, a_{\text {halo }}, h_{d}$ & $h_{d}$ \\
Model 4 & $\Sigma_{0, \text { young }}, \Sigma_{0, \text { old }}, a_{\text {halo }}, M_{\text {halo }} / M_{d}$ & $\Sigma_{0, \text { young }}$ \\
& & $\Sigma_{0, \text { old }}$ \\
Model 5 & $\Sigma_{0}, a_{\text {halo }}, h_{d}$ & $\Sigma_{0}, h_{d}$ \\
\hline Model 6 & $\Sigma_{0}, a_{\text {halo }}, h_{d}, \kappa_{0}$ & $\Sigma_{0}, h_{d}$ \\
Model 7 & $\Sigma_{0}, a_{\text {halo }}, \kappa_{0}$ & $\Sigma_{0}, h_{d}$ \\
\hline
\end{tabular}

\subsection{Results of the Modeling}

Fig. 7 shows examples of the chi-square minimization for some galaxies in our sample: a red one (upper right panel), a blue one (lower left case), a galaxy with bulge (lower right), and a galaxy in-between the red and blue superthins (upper left panel). The curves designate the contributions of disk (dashed curve) and dark halo (dash-dotted curve) to the rotation. The dotted curves show the total rotation curves from all galactic components. The solid curves show the results of the projection, i.e. the model observable rotation curves. The bullets with bars denote the observing data points and their uncertainties.

The modeling allows us to obtain parameters of stellar disks and dark halos for all our galaxies. The parameters are shown in Table 6. Fig. 8 demonstrates the resulting rotation curves for different models (the solid and dotted black lines) and the ranges of the rotation curves for the disk and halo components (shown with the shaded red and blue areas, respectively.) Fig. 8 also shows an example of similar resulting rotation curves after the edge-on projection obtained in different models while the disk and halo rotation curves can be significantly different. On average, the models improve best-fitting chi-square values with respect to Model 0. The least improving are Models 1 and 7 - their median chi-square difference from the Model 0 normalized by the latter is about $2 \%$, whereas the most improving are the Models 5 and 6 (about $50 \%$ of the median chi-square improvement.) We select the Model 6 for the further analysis because it has the best overall chi-square values for the models, on average.

The most consistent results between the models are obtained for the stellar disk scale length $h$ (when allowed to vary) and the dark halo mass $\log M_{h}$. The median value of the $h$ is greater by up to $3 \%$ in models $0,1,2,4, \& 7$, or lower by $1 \%$ in model 3 , with respect to model 6 . The median value of $\log M_{h}$ is less than $1 \%$ in models $0,1,3, \& 7$, and greater than $1 \%$ in models $2,4, \& 5$ with respect to model 6 . The disk central surface density is not farther than $10 \%$ from model 6 's values in models $0,3,5, \& 7$. This value differs from model 6 by more than $50 \%$ in model 4 . The halo scale $a_{\text {halo }}$ is within $10 \%$ from that in model 6 in all models except for models 2 and 4, where they are longer by up to $84 \%$. We notice that the halo scale shows a large overall scatter between the models. The halo-to-disk mass ratio is $30 \%$ lower in models $0,1, \& 6$, very consistent in models $3 \& 5$, and is greater by $140 \%$ in model 4 , with respect to model 6 . Not a surprise that the parameters closely related to measurable values - $h$ and $\log M_{h}$, are estimated the most reliably and therefore are consistent from model to model, while the dark halo structure parameter $a_{\text {halo }}$ varies significantly between our different models.

Having information about the gravitational potential of all galactic components and the surface density, we can assess the dynamical state of the disk as in Spitzer (1942) by estimating its vertical velocity dispersion at $2 h$ distance from the center as

$$
\sigma_{*}(2 h)=\left[\pi G \Sigma_{0} e^{-2} z_{0}\right]^{1 / 2} .
$$

Fig. 9 shows a relation between the central surface density and the vertical velocity dispersion estimated at two disk scale lengths. We check if the quality of fitting, in terms of the chi-square value, affects the results and the dependencies between the output parameters via dividing our objects by better- and worse-fitted samples. We divide the sample using the median chi-square value, and show results with different symbols in Fig. 9 and further figures in the paper. As it can be seen in Fig. 9, both better- and worse-fitted galaxies show the same trends, and our conclusions do not depend on the quality of fitting. Same conclusion can be inferred for the further figures. 

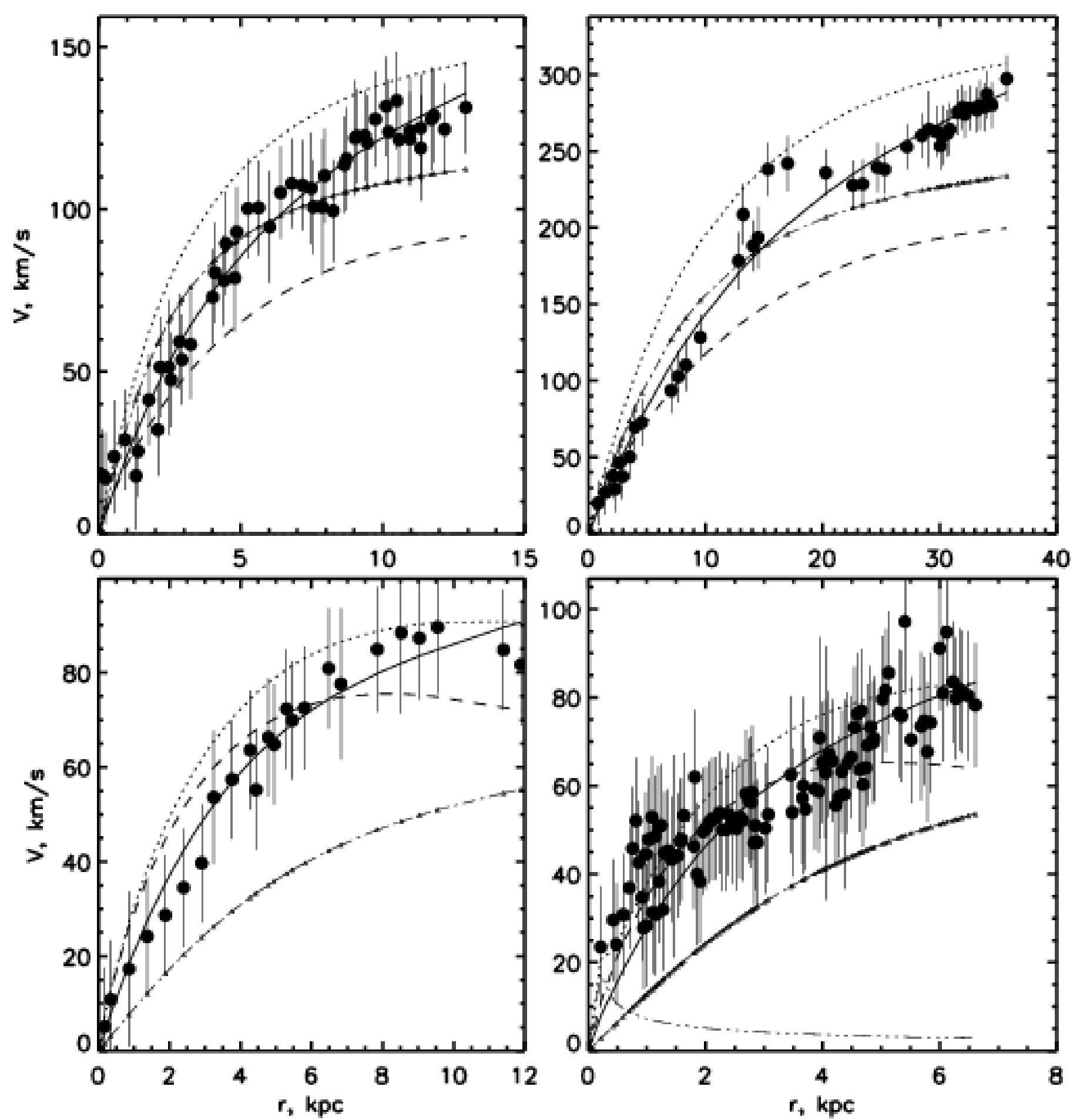

Figure 7. Examples of modeling for some types of our galaxies in the frames of the Model 6. The upper left panel demonstrates the modeling for a typical galaxy, EON_19.768_-0.139. The dash-dotted and dashed curves designate the contribution from the dark matter halo and stellar disk, respectively. The dotted curve is the true rotation curve from all galactic components. The solid curve is the true rotation curve after the edge-on projection. The bullets with error bars represent the observing rotation curve. The upper right panel shows the same curves for a red superthin galaxy, EON_149.150_20.646. The lower left panel is for a blue galaxy EON_51.832_3.920. The lower right panel shows the modeling for a galaxy with small bulge, EON_123.495_45.742. The bulge contribution is shown with the dash-doubledotted curve. 


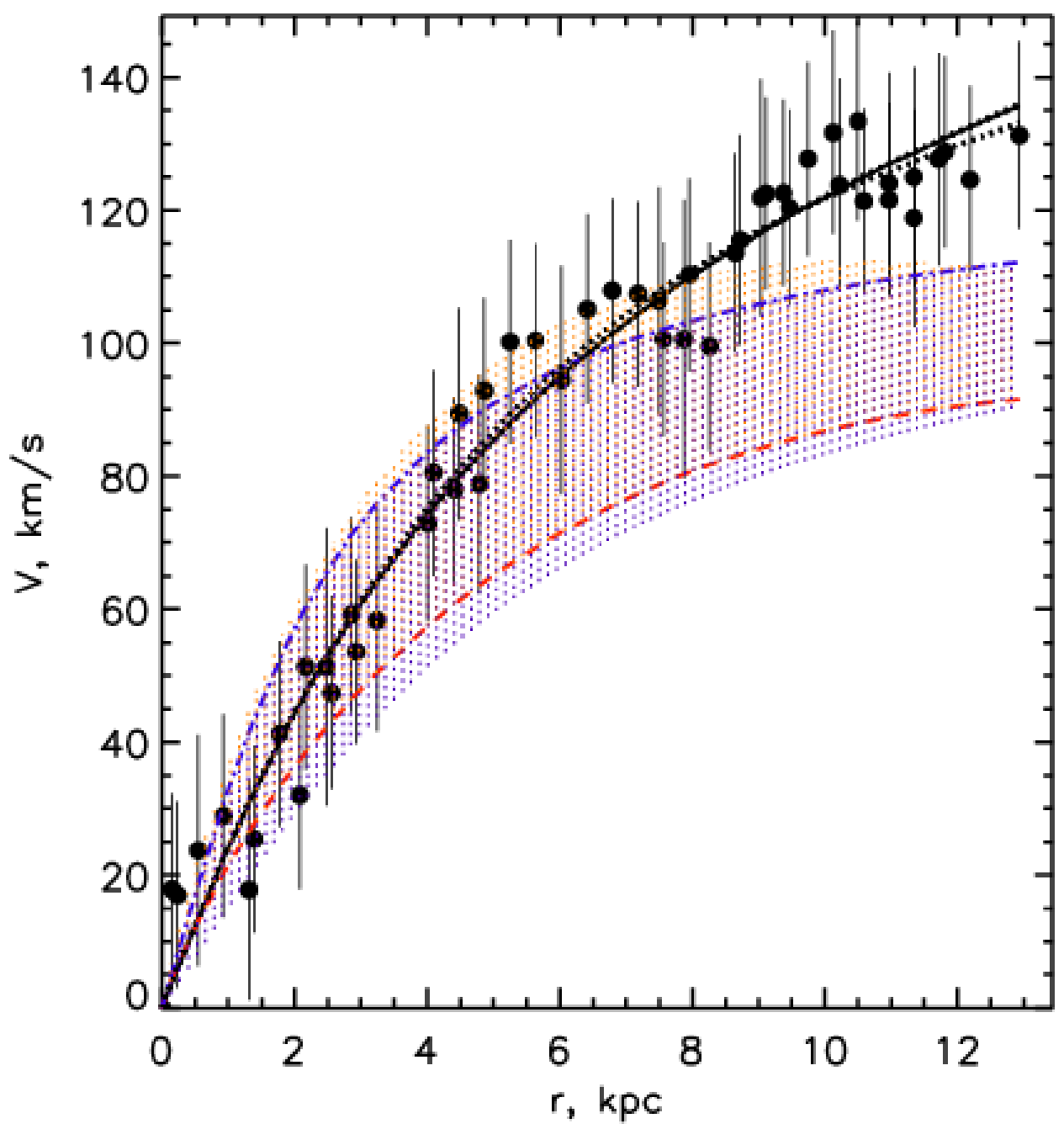

Figure 8. The ranges of the disk (red) and halo (blue) rotation curves between different models 0-7 are designated with the shaded areas for the galaxy EON_19.768_-0.139. The red dashed curve designates the disk-only rotation curve for Model 6, and the blue dash-dotted line is for the halo in the same model. The black solid curve denotes the sum rotation curve after the edge-on projection for the Model 6. All other models are designated with the dotted black curves. The bullets with error bars show the observing rotation curve.

We calculate the errors of resulting model output parameters for a typical galaxy EON_19.768_-0.139 shown in Fig. 7 via running the model 6 for it one hundred times with slightly changed input parameters. Each run we allow the input model parameters - stellar disk scales and velocities - to take random values according to their uncertainties from observations. We assume that the input errors are distributed normally. As a result, the modeling is run with slightly different parameters, and therefore produces different results. The typical uncertainties of resulting parameters are found as standard deviations of the output values.

Note that equation (12) suggests a correlation between the $\Sigma_{0}$ and $\sigma_{*}(2 h)$. We demonstrate Fig. 9 to show that the lowest surface density galaxies in our sample with $\Sigma_{0}<120 M_{\odot} \mathrm{pc}^{-2}$ have very low velocity dispersion under 10 $\mathrm{km} \mathrm{s}^{-1}$. It confirms that the thinnest disks have the lowest stellar densities, which also suggests that their stellar disks did not experience significant dynamical heating during the history of the galaxies, and kept their vertical dispersion close to that of the galactic gas through the galactic history. 


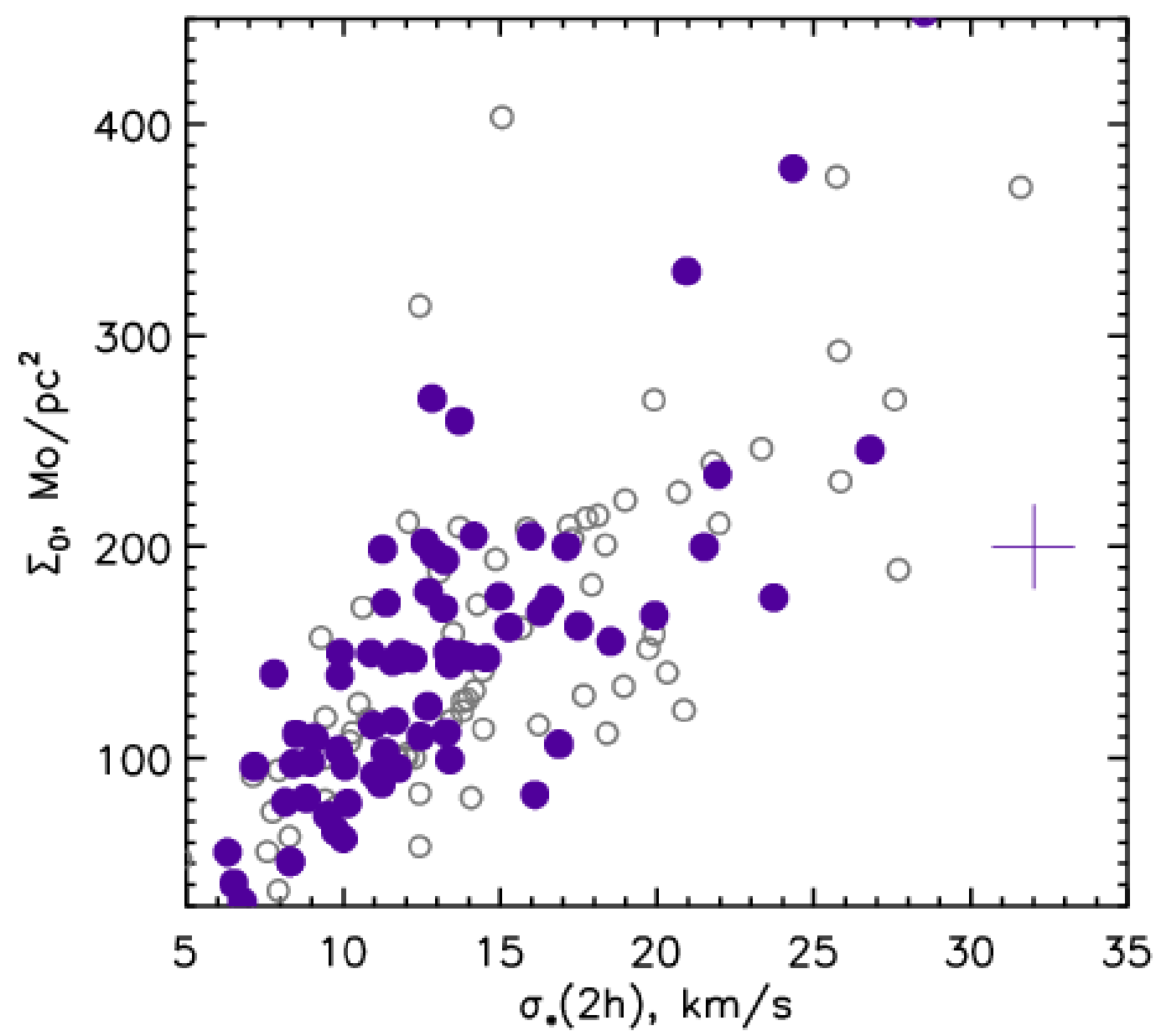

Figure 9. The disk $\Sigma_{0}-\sigma_{*}(2 h)$ diagram. The vertical velocity dispersion at two disk scales from the center $\sigma_{*}(2 h)$ is estimated via equation (12). The grey circles represent the worse than average fitting (chi-squared is greater than the median of the sample), while the blue bullets designate our galaxies with good fitting. The blue cross demonstrates the typical error bars along the axes.

If we replace the surface density with galactic colors as in Fig. 10, it is clear that the most dynamically under-evolved galaxies are among the blue subsample with the colors $(r-i)_{0}<0.24$ mag. It is also interesting to notice that the vertical velocity dispersion falls below $10 \mathrm{~km} \mathrm{~s}^{-1}$ in the galaxies whose disk central surface density goes below the average dark halo surface density $\left(90-110 M_{\odot} \mathrm{pc}^{-2}\right)$ inferred by Kormendy \& Freeman (2004); Di Paolo et al. (2019), i.e. the dark halo dominates in them everywhere by the surface density. On a contrary, the reddest galaxies in the sample indicate rather high vertical velocity dispersion with respect to the gas, a few tens $\mathrm{km} \mathrm{s}^{-1}$.

Although the dark matter halo parameters - mass and scale - have different absolute values between the models, we can notice a common behavior of these parameters considered in the relative sense. We observe the following common tendencies. While a correlation between the dark halo mass $M_{h}$ and the maximum rotational velocity is expected, the halo scale length $a_{\text {halo }}$ shows the inverse dependence of the $V_{\max }$, and also of the color, see Fig. 11. It is notable to mention that the blue galaxies tend to have extended dark halos, and the halo-to-disk scale ratios are high for the blue STGs as well. On a contrary, the red STGs have rather short-scaled dark halos (small $a_{\text {halo }} / h_{d}$ ratios), which is in agreement with results of numerical simulations by Di Paolo et al. (2019).

The galaxies with high $V_{\max }$ also have compact halos, whereas the low rotation curve maxima follow the rarefied halos (Fig. 12) in a combination with LSB disks, as in simulations by Maccio et al. (2007). 


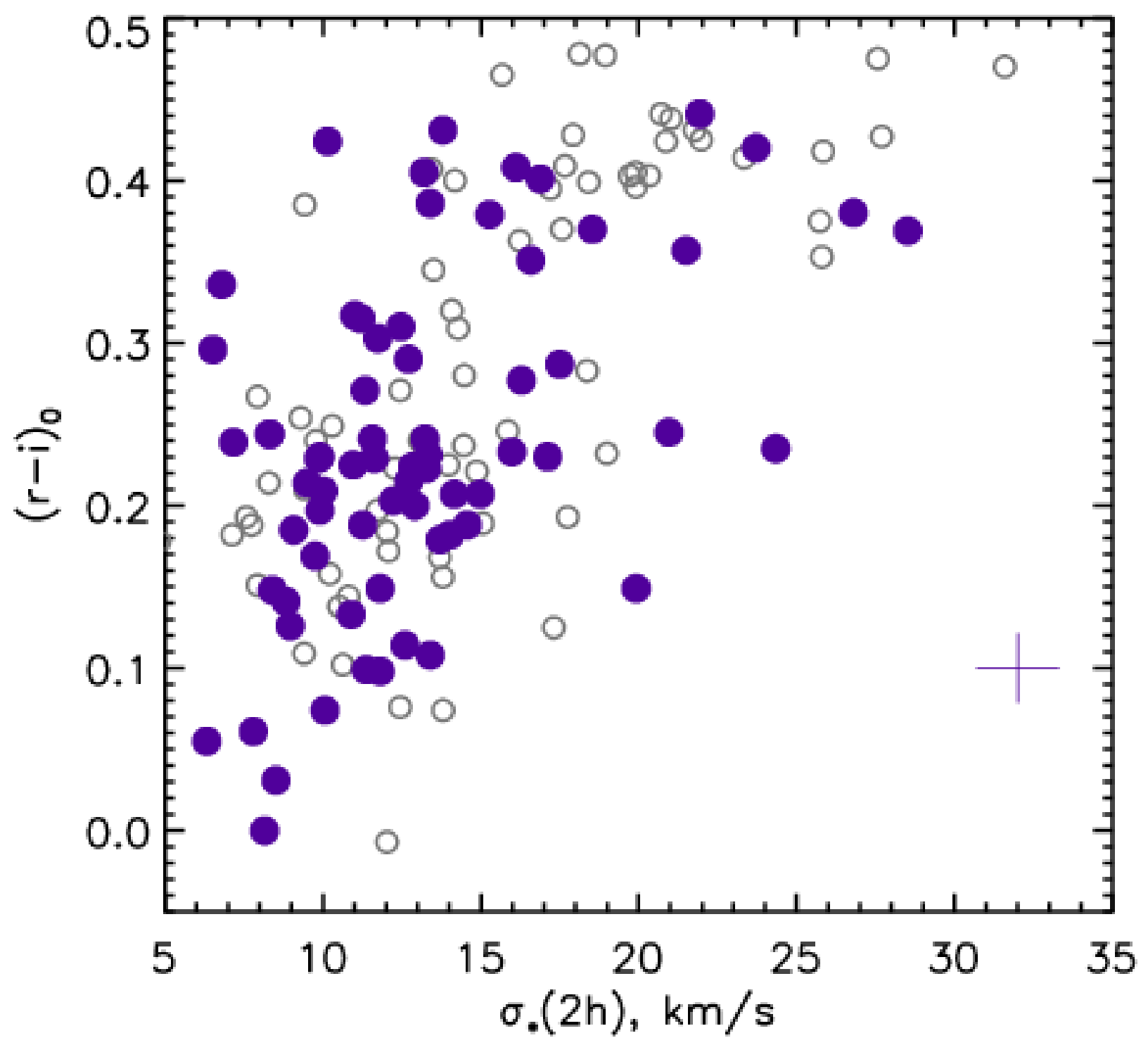

Figure 10. The color - stellar velocity dispersion diagram. The designations are kept as in Fig. 9.

The masses of dark halos correlate with the colors $(r-i)_{0}$, and the most massive halos are observed in the reddest STGs in our sample, see Fig. 13. The halo-to-disk mass ratio, which reveals the dark-to-light mass in the galaxies, does not show a clear systematic variation with the galactic color (Fig. 13) or with $V_{\max }$.

\subsection{Modeling with the Observed Disk Thickness Radial Distribution}

A way to include the observed radial distribution of the stellar disk thickness into the modeling was presented by Narayan \& Jog (e.g. 2002); Banerjee \& Jog (e.g. 2008); Banerjee \& Bapat (e.g. 2017). We follow it, and start with the Poisson equation for the thin disk:

$$
\frac{\partial^{2} \Psi_{s}}{\partial z^{2}}+\frac{\partial^{2} \Psi_{g}}{\partial z^{2}}+\frac{\partial^{2} \Psi_{h}}{\partial z^{2}}=4 \pi G\left(\rho_{s}+\rho_{g}+\rho_{h}\right)
$$



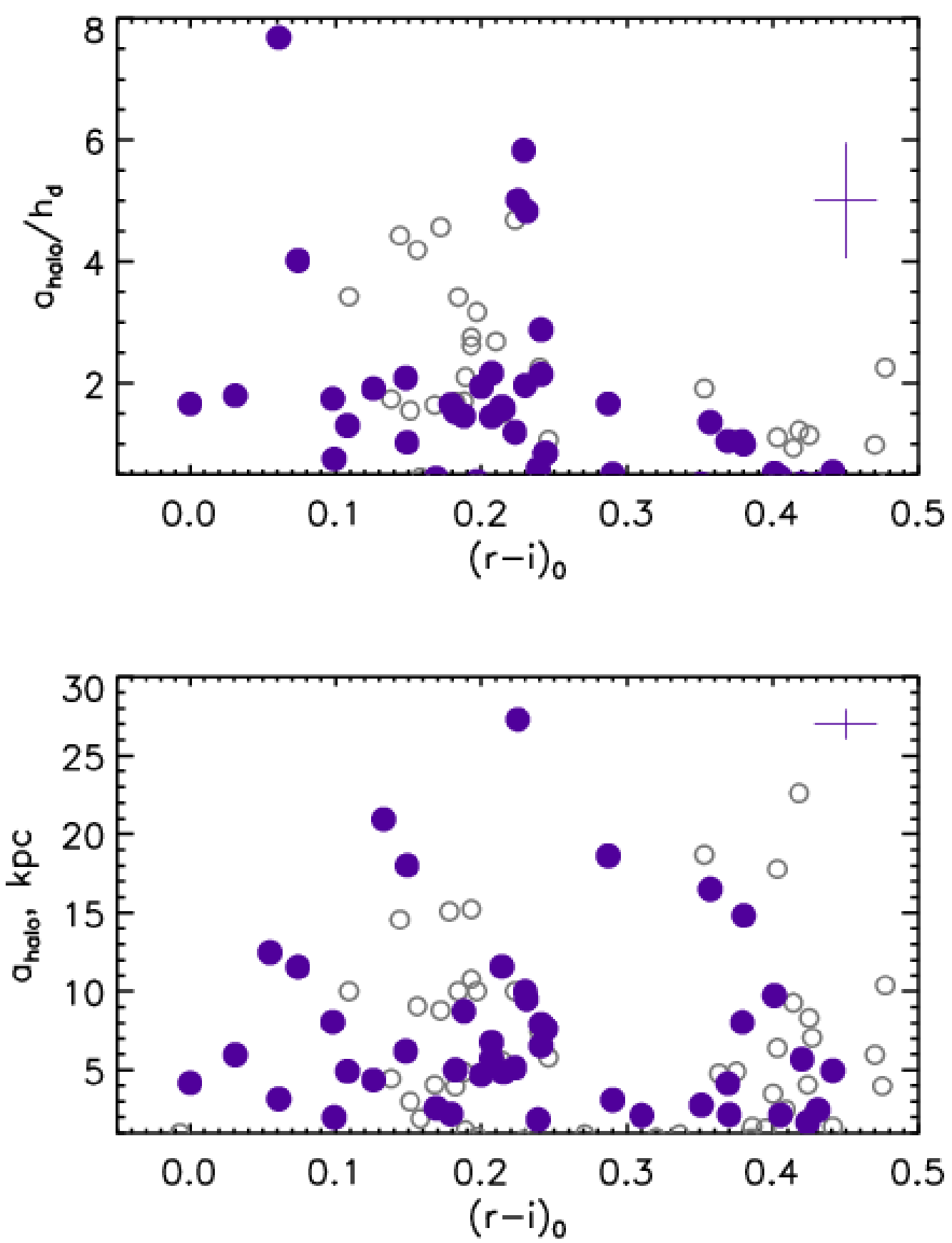

Figure 11. Upper panel: the dark halo scale normalized by the disk scale length $a_{\text {halo }} / h_{d}$ versus the galactic color $(r-i)_{0}$.

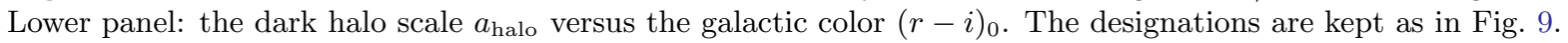

where the subscripts "s", "g", and "h" refer to the stars, gas, and halo, respectively. We combine equation (13) with the hydrostatic equilibrium equation in the vertical direction

$$
\frac{<\left(c_{z}\right)_{i}^{2}>}{\rho_{i}} \frac{\partial \rho_{i}}{\partial z}=K_{s}+K_{g}+K_{h}
$$




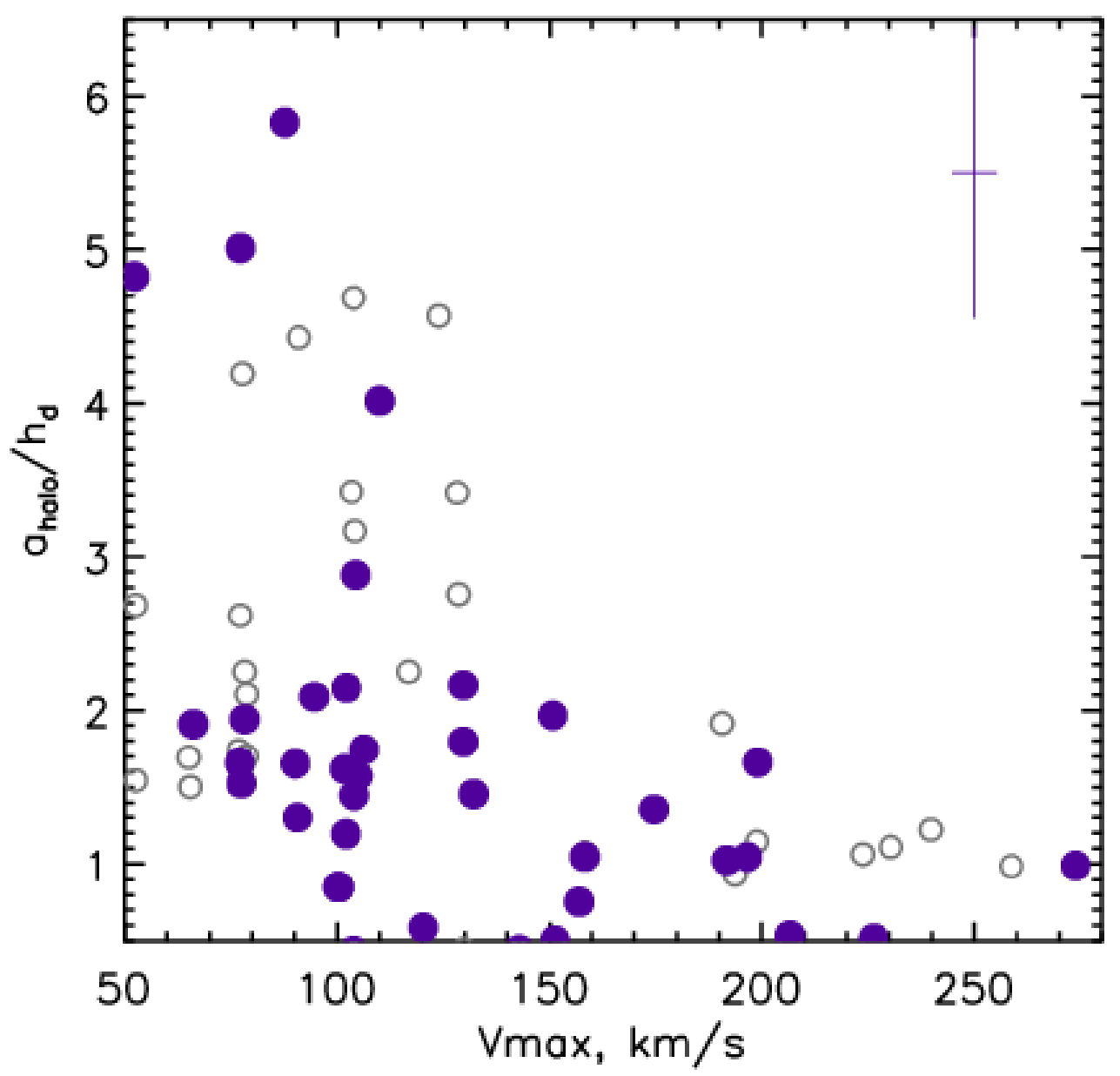

Figure 12. The dark halo scale $a_{\text {halo }}$ - the maximum rotation velocity $V_{\max }$ diagram. The designations are kept the same as in Fig. 9.

where $K_{i}=\partial \Psi_{i} / \partial z$, and the " $i$ " subscript refers to either stars or gas or dark halo, and $\left\langle\left(c_{z}\right)^{2}\right\rangle$ is the root mean square of the vertical velocity. By combining equations (13) and (14) we obtain a system of equations for each component. We assume that the density distributions in the halo and disks follow equations $(5,11,6)$ and use equations for the gravitational potentials by van der Kruit \& Searle (1982); Narayan \& Jog (2002); van der Kruit (1988). Since we intend to compare the resulting vertical disk scale with observed $z_{0}$ estimated for the case described by equation (5), we substitute the functional form to equations (13) and (14) and solve them on iterative way. Given the same functional forms of the galactic components, the rotation curve fitting was kept identical to §4.1.

The obtained model disk thickness $z_{0}$ is integrated along the line of sight in the edge-on, dusty disk with equations analogous to (11) and (10). The resulting model disk thickness is compared to the observed ones along the radius in same manner as it was done to the velocities. We find the best-fit parameters by minimizing the chi-square of differences between observed and model parameters. The minimized chi-square is the sum of chi-squares obtained for the velocity and disk thickness separately. Note that the scale height distribution provides twice as many data points as the rotation curve for the minimization. To equalize contributions of the two factors to the overall chi-square, we attempted to multiply the chi-squares by the number of data points, as well as by the squared number of data points. The resulting best-fit parameters did not change significantly. We perform this modeling for the galaxy UGC 7321 . Figure 14 shows results of simultaneous fitting of the rotation curve and thickness distribution. The latter is estimated from Bizyaev et al. (2014), where individual vertical cuts along the minor axis were analyzed based on SDSS images in the $r$ band. 

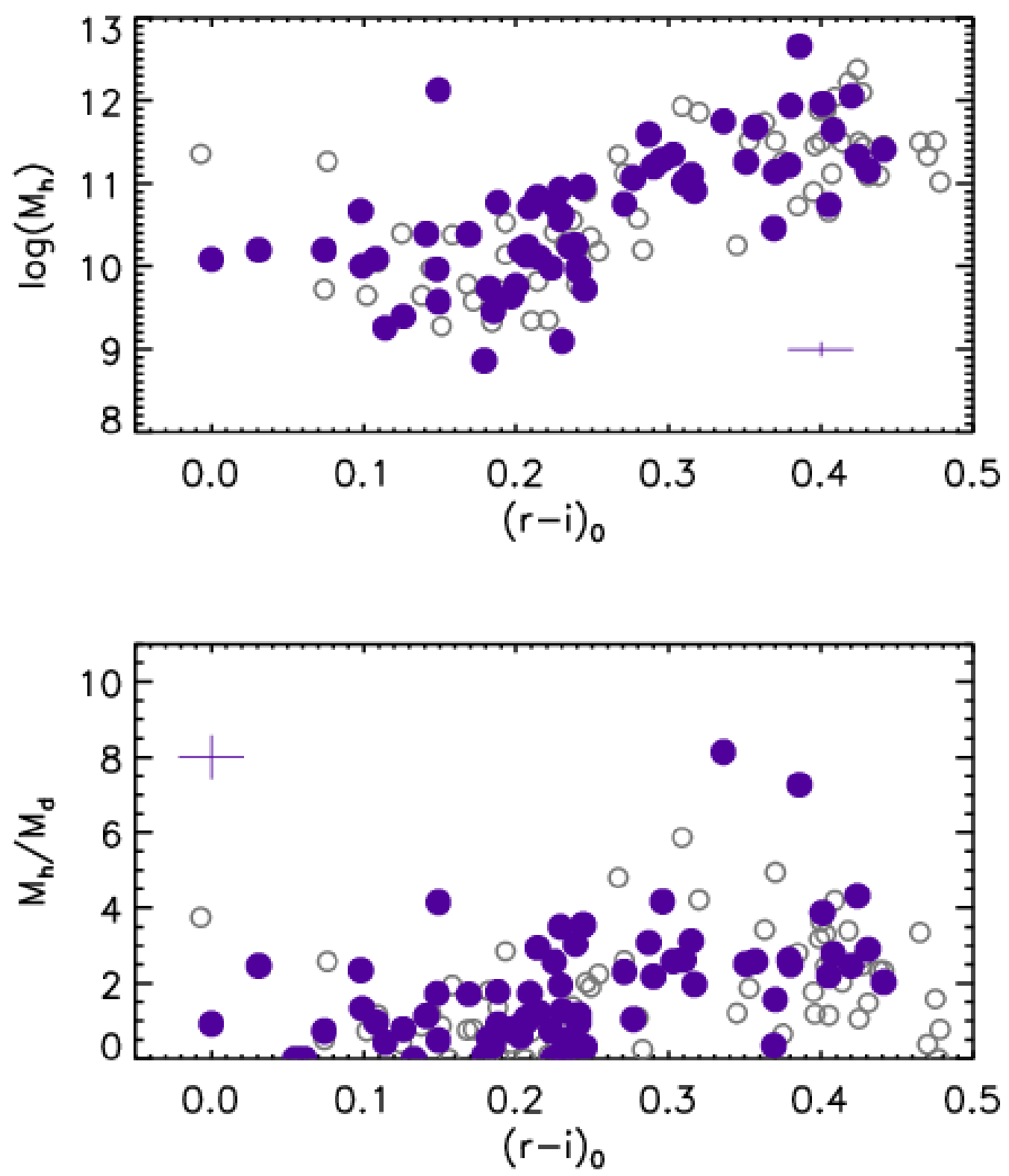

Figure 13. The M_halo - (r-i) and M_halo / Md- (r-i) panels. The designations are kept the same as in Fig. 9.

We develop the model with a set of constraints similar to the Model 6 described above. Two additional constraints are the use of the halo-to-disk ratio and the gas disk central surface brightness as free parameters. The former parameter is introduced because we don't use equation (7) anymore. Note that the latter parameter is highly degenerated with the stellar surface density because of the similar assumed functional form for the gas radial distribution. In the frames of the assumptions the "disk surface density" can be referred to the stars and gas summed together. As a result, we obtained qualitatively similar parameters in our modeling here and in the Model 6: this galaxy indicates a rather small halo-to-disk scale ratio and high halo-to-disk mass. Qualitatively, these results also agree with the pseudo-isothermal halo case in the modeling by Banerjee \& Bapat (2017).

\section{DISCUSSION}



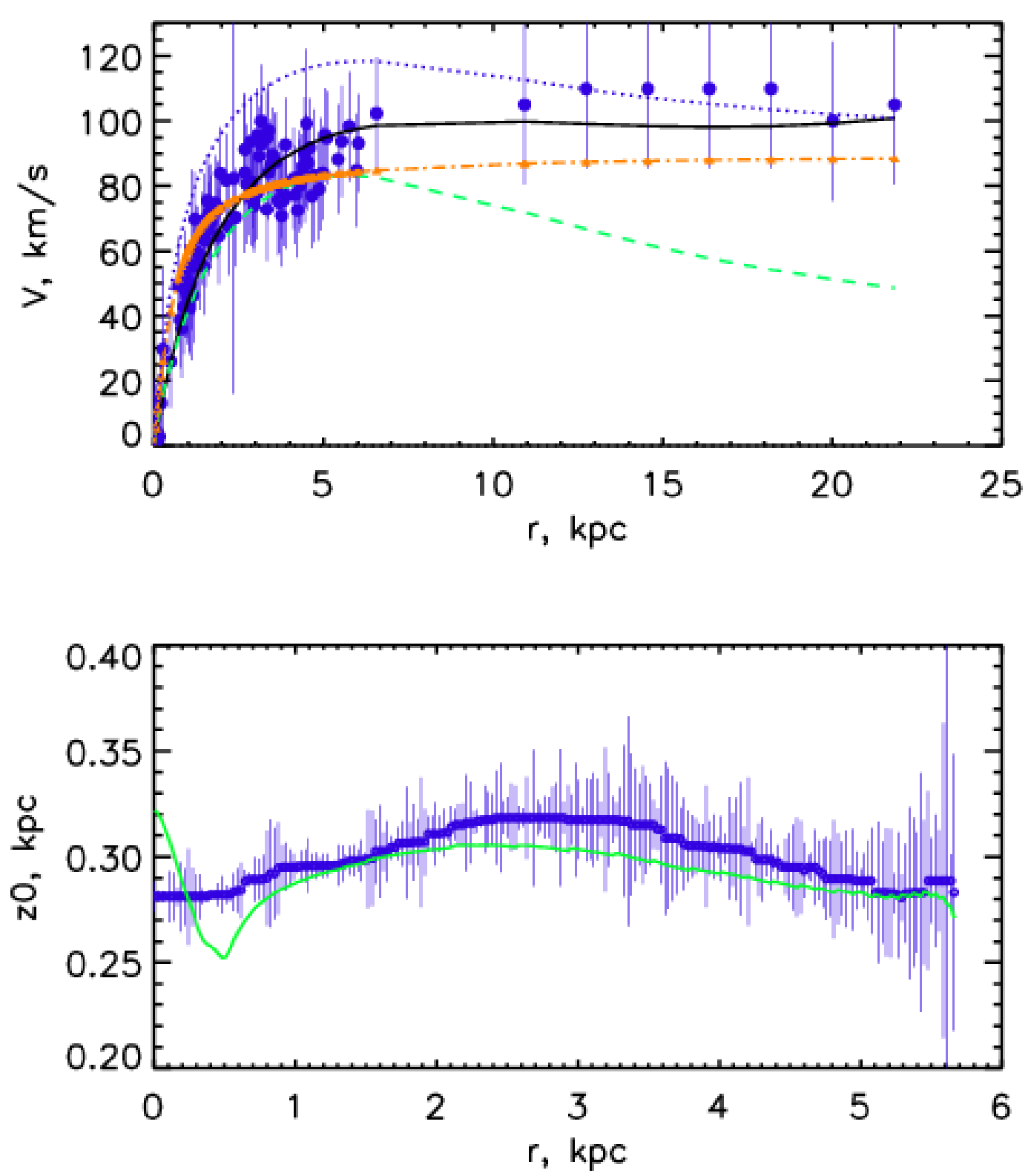

Figure 14. Results of simultaneous modeling of the rotation curve and the disk thickness radial distribution for the galaxy UGC 7321. The upper panel has the same designations as in Figure 7. Our ionized gas rotation curve was updated with the rotation measurements from Uson \& Matthews (2003) in the outer disk, beyond $7 \mathrm{kpc}$ from the center. The lower panel shows the observed (blue dots with error bars) and model (green curve) disk thickness radial distribution.

Our observations and modeling demonstrate a noticeable dependence of STGs properties of their color. The color $(r-i)_{0}$ correlates with the rotation curve maximum (Fig. 4) and with estimated vertical velocity dispersion of stars (Fig. 10). The dark halo scale normalized by the disk scale length is shorter in the red STGs (Fig. 10), although a majority of the blue and almost all red STGs have the $a_{\text {halo }} / h_{d}$ ratio less than 2 , in agreement with Banerjee \& Jog (2013); Banerjee \& Bapat (2017). Most of considered galaxies are dark matter dominated, and their dark halo mass also correlates with galactic color (Fig. 13), as it would be expected from Fig. 4.

Results of our modeling confirm the conclusion by B17 of a non-uniformity of superthin galaxies. The blue STGs with $(r-i)_{0} \leq 0.24$ mag look dynamically under-evolved: Fig. 10 shows that their stellar vertical velocity dispersion 
is close to that expected in the star forming galaxies (see e.g. Kennicutt (1989); Mogotsi et al (2015)), which has been noticed for two superthin galaxies (van der Kruit et al. 2001). At the same time, the central surface density of disks in these galaxies is very low, under $120 M_{\odot} \mathrm{pc}^{-2}$ (Fig. 9). The blue STGs preserved themselves from different internal and external factors that increase the vertical stellar velocity dispersion: mergers (Walker et al. 1996; Velazquez \& White 1999; Qu et al. 2011) and growth of internal non-axisymmetric instabilities (Barbanis \& Woltjer 1967; Khoperskov et al. 2010; Saha 2014; Grand et al. 2016). As noticed by B17, the blue STGs stay away from busy cosmological environment, like filaments, which is also typical for LSB galaxies (Mo et al. 1994; Rosenbaum \& Bomans 2004; Kautsch 2009; Rosenbaum et al. 2009).

The red STGs indicate a higher stellar velocity dispersion (but still less than in Milky Way-like galaxies). Their disks look thin mostly due to their large disk scale length. In this sense they resemble the giant LSB galaxies, whose disks may form by continuous accretion events rather than a one-epoch assembly (Hagen et al. 2016).

In both cases a massive (in the sense of the high dark-to-light mass ratio) dark halo helps suppress the internal instabilities and keeps the disks thin (Zasov et al. 1991; Bizyaev \& Mitronova 2002; Kautsch 2009; Khoperskov et al. 2010; Ghosh \& Jog 2014). A central mass concentration like a massive compact bulge or compact halo helps stabilize the stellar disks against non-asymmetric instabilities and prevents the disks from increasing their thickness (Sotnikova \& Rodionov 2005, 2006; Kurapati et al. 2018).

The role of rotation in shaping the STGs should be essential: Jadhav \& Banerjee (2019) noticed that the spin angular momentum parameter $\lambda$ (Peebles 1969; Dalcanton et al. 1997; Bullock et al. 2001) for stars is significantly higher in the STG with respect to the normal galaxies, similar to LSBs (Maccio et al. 2007; Perez-Montano \& Cervantes Sodi 2019). In addition to a relatively high mass, a parameter of the internal structure of dark halo, such as the halo scale length, is assumed to be a key factor that shapes and preserves the STGs. Thus, Banerjee \& Jog (2013); Kurapati et al. (2018) found that the halo scale is short (under two stellar disk scale length) in a few STGs, whereas the regular high surface brightness galaxies have longer dark halo scales (Gentile et al. 2004; Narayan et al 2005; Banerjee \& Jog 2008; Khoperskov et al. 2010). Di Paolo et al. (2019) found that the halo-to-disk scale ratio $R_{\text {halo }} / R_{\text {disk }}$ anti-correlates with the dark halo compactness. Our results are in agreement with the short dark halo scale in most of STGs: Fig. 11 shows that more than a half of galaxies have $a_{\text {halo }} / h_{d}$ under 2 . At the same time, the blue STGs in our sample show higher $a_{\text {halo }} / h_{d}$ values (Fig. 11 and 12), which suggests that they have more compact dark halos, according to Di Paolo et al. (2019). This also is in agreement with simulations by Jiang et al. (2019). Note also that the least scale ratios $a_{\text {halo }} / h_{d}$ correspond to the red STGs (Fig. 11). They also have massive halos and highest dark-to-luminous mass ratios (Fig. 13).

We employ the definitions of the spin angular momentum $\lambda$ from Bullock et al. (2001); Jiang et al. (2019) to estimate the parameter $\lambda_{\text {gal }}$ for the disks in our galaxies. The mean $\lambda_{\text {gal }}$ for the disk is 0.078 , which is some three times higher than in typical galaxies (Jiang et al. 2019). The blue and red STG groups show similar mean $\lambda_{\text {gal }}$.

Dalcanton et al. (2004) noticed that small galaxies with the rotational velocity under $120 \mathrm{~km} \mathrm{~s}^{-1}$ show a lack of the visible dust layer. Our Figures 4 and 10 show that small STGs bluer than $(r-i)_{0}=0.24$ mag have their rotational velocities under $120-125 \mathrm{~km} \mathrm{~s}^{-1}$ and the vertical stellar velocity dispersion close to that in the gas. It means their disks of stars and dust have similar thickness, therefore the dust layer cannot be clearly seen, which naturally explains the transition from galaxies with organized dust lanes to those without them at this specific value of the rotational velocities.

\subsection{Limitations of the Models and the Further Modeling}

Detailed modeling of galaxies is a complex and time consuming task. We decided for an efficient, powerful, and established approach. Our modeling is data driven by adapting a specific vertical distribution of stellar density following van der Kruit \& Searle (1981); Bottema (1993). This enables the active comparisons of derived structural parameters in large catalogs, such as Bizyaev et al. (2014). The exact solution of Poisson and hydrostatic vertical equilibrium equations (Bahcall 1984) generates corrections to the disk vertical density profile in eq. (5) for halo gravitation. As shown by Bahcall (1984), this correction is small.

Solving the Poisson and hydrostatic equilibrium equations as in Narayan \& Jog (2002); Banerjee \& Jog (2013); Aditya \& Banerjee (2020) and comparing the resulting disk thickness radial distribution with observations has several more degrees of freedom to explore: from testing different density distributions in the dark halo to introducing the density-luminosity constraints. We postpone a more complicated analysis for a future publication. In this work we describe our data-driven procedure in eqs. (5-11) that employs only one scaling relation described by eq. (7). It is 
conceived from results of N-body simulations, and proved on both HSB and LSB galaxies therein. Note that two our models developed above do not use eq. (7) at all. Our results show that our controlled modeling facilitates the use in our large set of galaxies with existing catalog data. To further investigate the effectiveness of our model, we plan to compare the different approaches in a specialized upcoming article.

\subsection{Comparison With Published Results}

Our sample has three STGs studied in the past: UGC 7321, IC 2233, and UGC 9242. Besides the first object investigated in detail (Matthews 2000; Uson \& Matthews 2003; Banerjee \& Bapat 2017), IC 2233 was modeled by Banerjee \& Bapat (2017). Spectra of UGC 9242 were published by Goad \& Roberts (1981). One more galaxy observed by us, UGC 711, was mentioned by Mendelowitz et al. (2000), but the publication does not have the actual data available for comparison.

IC 2233: Banerjee \& Bapat (2017) employs the Salo et al. (2015) two-disk fitting to the Spitzer NIR photometry for this galaxy. Our optical and NIR disk scales are close to the published data. Our ionized gas rotation curve maximum is close to that obtained by Matthews \& Uson (2008). Our rotation curve is limited by the spectrograph's long slit size, and its spacial extension is 30 per cent smaller than the published HI curve. This galaxy indicates a non-standard rotation curve affected by small-scale instabilities (Matthews \& Uson 2008b). Our modeling is not conclusive, and the methods of modeling applied by us yield either extremely long or extremely short halo scales. The rotation curve shape is not smooth for this galaxy, and a custom-made model with trials of different mass profiles for the bulge and disk for this galaxy is required to reproduce its rotation curve better.

UGC 7321. A state-of-art modeling of the prototype superthin galaxy UGC 7321 was performed by Matthews (2000); Uson \& Matthews (2003); Matthews \& Wood (2001); Banerjee \& Jog (2013). We use a shorter distance to the galaxy with respect to Matthews (2000). Our optical scale length is slightly longer than that from Matthews (2000) (56 versus 43 arcsec), while the $H$ band scale length is shorter (21 versus 41 arcsec). The scale heights we use are comparable to the published if converted to the exponential density distribution in the vertical direction. The HI rotation curve obtained by Uson \& Matthews (2003) is twice as more extended than our H $\alpha$ curve, but the latter reaches the distance where the curve becomes flat.

UGC 9242: the major axis spectrum was published by Goad \& Roberts (1981), and it is in agreement with the spectra we obtained. Our rotation curve is slightly more extended (by 10 per cent) than the published.

\section{SUMMARY}

We obtain spectra of 138 superthin galaxies with the resolution of R 5000 and perform NIR observations of 18 STGs from that sample on the 3.5m telescope at the Apache Point Observatory. The combination of our spectroscopy, NIR photometry, published NIR data (Bizyaev et al. 2020), and SDSS photometry allows us to develop a set of constrained models taking into account the vertical structure of stellar disks. This enables us to study the dark matter halo parameters and dynamical status in the largest, uniform sample of superthin galaxies.

We find that the sample of superthin galaxies is not uniform, and can be subdivided by the blue, $(r-i)_{0} \leq 0.24$ mag, and red, $(r-i)_{0}>0.24 \mathrm{mag}$, STGs. The blue superthin galaxies are mostly dynamically under-evolved. Their stellar vertical velocity dispersion is as low as that in the gas. The red STGs show higher vertical velocity dispersion, in combination with a very large disk scale length.

The surface brightness in the disks of the superthin galaxies is constrained by the minimum vertical stellar velocity dispersion that cannot be lower than that of the galactic star forming gas. In addition, there may be a limiting central surface density, which is of the order of $50 M_{\odot} \mathrm{pc}^{-2}$ from our simplified toy model.

Most of our STGs are dark matter dominated. Their rotational velocity and dark halo mass correlates with galactic color. The blue STGs also have less compact dark halos than the red STGs, whereas the galaxies in both color groups have their halo-to-disk scales ratios under two.

\section{ACKNOWLEDGEMENTS}

The project is partly supported by RScF grant 19-12-00145. VPR and AVA acknowledge the support by RFBR grant 19-32-50129 for an analysis of the photometry of the superthin galaxies. The database support was provided in framework of RFBR grant 19-32-90244.

We thank anonymous referee whose comments and valuable suggestions improved the paper. Based on observations obtained with the Apache Point Observatory 3.5-meter telescope, which is owned and operated by the Astrophysical Research Consortium. 
Table 6. Parameters of the galaxies obtained in Model 6. The columns show the name, central surface density (in $M_{\odot} \mathrm{pc}^{-2}$ ), fitted disk scale length (kpc), halo scale (kpc), dark halo mass, disk mass, and the best fitting chi-square value.

\begin{tabular}{lllllll}
\hline Name & $\Sigma_{0}$ & hfit & ahalo & $\log M_{\text {halo }}$ & $\log M_{d}$ & chi**2 \\
\hline \hline EON_105.825_13.464 & 162. & 7.67 & 8.03 & 11.22 & 10.80 & $3.215 \mathrm{e}+00$ \\
EON_11.160_-11.189 & 222. & 4.37 & 45.19 & 10.27 & 10.43 & $8.352 \mathrm{e}+00$ \\
EON_115.887_31.535 & 147. & 2.28 & 49.96 & 9.58 & 9.89 & $1.000 \mathrm{e}+00$ \\
EON_116.146_18.328 & 269. & 4.83 & 0.14 & 10.65 & 10.60 & $9.946 \mathrm{e}+00$ \\
EON_118.918_28.743 & 122. & 2.16 & 9.05 & 0.00 & 9.73 & $5.063 \mathrm{e}+00$ \\
$\ldots$ & Table is published in its entirety & \\
\hline \multicolumn{6}{c}{ in the electronic edition. ... } \\
\hline
\end{tabular}

We acknowledge the usage of the HyperLeda database ${ }^{5}$ (Makarov et al. 2014). This research has made use of the NASA/IPAC Extragalactic Database (NED) which is operated by the Jet Propulsion Laboratory, California Institute of Technology, under contract with the National Aeronautics and Space Administration.

\section{REFERENCES}

Aditya, K. \& Banerjee, A. 2020, arxiv:2002.09198

Alam, S., Albareti, F. D., Allende Prieto, C. et al. 2015, ApJS, 219, 12

Angus, G., Gentile, G., Swaters, R. et al. 2015, MNRAS, 451, 3551

Bahcall, J. 1984, ApJ, 276, 156

Banerjee A. \& Jog C. J., 2008, ApJ, 685, 254

Banerjee, A., Jog, C., \& Matthews, L. 2009, ASP Conf. Ser., 407, 99

Banerjee, A. \& Jog, C. 2013, MNRAS, 431, 582

Banerjee, A. \& Bapat, D. 2017, MNRAS, 466, 3753

Barbanis, B. \& Woltjer, L. 1967, ApJ, 150, 461

Beijersbergen, M., de Blok, W. J. G., \& van der Hulst, J. M. 1999, A\&A, 351, 903

Bell, E., McIntosh, D., Katz, N. and Weinberg, M. 2003, ApJS, 149, 289

Bianchi, S. 2007, A\&A, 471, 765

Binney J. \& Tremaine S., 1987, Galactic Dynamics.

Princeton, NJ, Princeton Univ. Press

Bizyaev, D. \& Kajsin, S. 2004, ApJ, 613, 886

Bizyaev , D. \& Mitronova, S. 2002, A\&A, 100, 200

Bizyaev , D. \& Mitronova, S. 2009, ApJ, 702, 1567

Bizyaev, D., Kautsch, S., Mosenkov, et al. 2014, ApJ, 787, 24 (EGIS)

Bizyaev, D., Kautsch, S., Sotnikova, N. Ya., et al. 2017, MNRAS, 465, 3784 (B17)

Bizyaev, D., Tatarnikov, A., Shatsky, N., et al. 2020, Astr. Nachr. 341, 314

Bogelman, M. \& Shlosman, I. 2009, ApJL, 702L, 5

${ }^{5}$ http://leda.univ-lyon1.fr
Bosma, A., Byun, Y., Freeman, K. C. \& Athanassoula, L. 1992 ApJ, 400, L21

Bottema, R., 1993, A\&A, 275, 16

Bournaud, F., Jog, C. J., \& Combes, F. 2007, A\&A, 476, 1179

Bullock, J. S., Kolatt, T. S., Sigad, Y., et al. 2001, MNRAS, 321, 559

Bullock, J. S., Stewart, K. R., \& Purcell, C. W. 2008, IAU Symp., 254, 1

Charlot, S. \& Longhetti, M. MNRAS, 323, 887

Clark, P. C. \& Glover, S. 2014, MNRAS, 44, 2396

Courteau, S. 1997, AJ, 114, 2402

Courteau, S., Dutton, A., van den Bosch, F., et al. 2007, ApJ, 671, 203

Courtois, H. M., Tully, R. B., Fisher, J. R. et al. 2009, AJ, 138,1938

Dalcanton, J. J., Spergel, D. F., \& Summers, F. J. 1997, ApJ, 482, 659

Dalcanton, J. J., Yoachim, P., \& Bernstein, R. A. 2004, ApJ, 608, 189

Dehnen, W. \& Binney, J.J. 1998, MNRAS, 298, 387

Di Paolo,C., Salucci, P. \& Erkurt, A. 2019, MNRAS, 490, 5451

D’Onghia E., Burkert A., Murante G., Khochfar S., 2006, MNRAS, 372, 1525

Efstathiou, G. 2000, ApJ, 317, 697

Gentile G., Salucci P., Klein U., Vergani D., Kalberla P., 2004, MNRAS, 351, 903

Gerssen, J., Kuijken, K., \& Merrifield, M. 2000, MNRAS, 317,545

Ghosh S., Jog C. J., 2014, MNRAS, 439, 929 
Giovanelli, R. Avera, E. \& Karachentsev, I. D. 1997, AJ, 114,122

Graham, A. 2002, MNRAS, 334, 721

Grand, R. J. J., Springel, V., Gomez, F. A. et al. 2016, MNRAS, 459, 199

Goad, J. W., Roberts, M. S. 1979, BAAS, 11, 668

Goad, J. W., Roberts, M. S. 1981, ApJ, 250, 79

Guiderdoni, B. 1987, A\&A, 172, 27

Hagen, L. M. Z., Seibert, M., Hagen, A. et al. 2016, ApJ, 826,210

Hall, M., Courteau, S., Dutton, A., et al. 2012, MNRAS, 425, 2741

Haynes, M. P., Giovanelli, R., Kent, B. R. 2018, ApJ, 861, 49

Impey, C. \& Bothun, G. 1989, ApJ, 341, 89

Jiang, F., Dekel, A., Kneller, O. et al. MNRAS, 488, 4801

Jadhav, V. \& Banerjee, A. 2019, arXiv:1906.10039v1

Jarrett, T. H., Chester, T., Cutri, R., et al. 2003, AJ, 125, 525

Jimenez, R., Verde, L. and Oh, S. P. 2003, MNRAS, 339, 243

Jog, C. \& Solomon, P. 1984, ApJ, 276, 127

Karachentsev, I. D., Karachentseva, V. E., Parnovskij, S. L. 1993, Astronomische Nachtichten, 314, 97

Karachentsev, I. D., Karachentseva, V. E., Kudrya, Yu. N., et al., 1999, Bull. Spec. Astr. Obs., 47, 5

Karachentsev, I. D., Karachentseva, V. E. \& Kudrya, Yu. N., 2016, arXiv:1605.03734

Kasparova, A., Saburova, A., Katkov, I., et al. 2014, MNRAS, 437, 3072

Kautsch, S. J., Grebel, E. K., Barazza, F. D., \& Gallagher, J. S., III 2006, A\&A, 445, 765

Kautsch, S. J., Gallagher, J. S., \& Grebel, E. K. 2009, Astronomische Nachrichten, 330, 1056

Kautsch, S. J. 2009, PASP, 121, 1297

Kazantzidis, S., Bullock, J. S., Zentner, A. R., Kravtsov, A. V., et al. 2008, ApJ, 688, 254

Kazantzidis, S., Zentner, A. R., Kravtsov, A. V., Bullock, J. S. et al. 2009, ApJ, 700, 1896

Kennicutt, R. C. 1989, ApJ, 344, 685

Khoperskov, A., Zasov, A., \& Tyurina, N. 2003, Astron. Reports, 47, 357

Khoperskov, A., Bizyaev, D., Tiurina, N., \& Butenko, M. 2010, Astronomische Nachrichten, 331, 731

Kormendy, J. 1983, in Morphology and dynamics of galaxies, ed. L. Martinet \& M. Mayor (Genf: SSAA), Saas-Fee Advanced Course, 12, 113

Kormendy,J. \& Freeman,K.C. 2004, IAU Symposium ,Sydney ,Astron. Soc. of the Pacific, 220, 377
Kormendy, J. \& Fisher, D. B. 2005, RMXAA (Conf. Ser.), 23, 101

Kormendy,J. \& Freeman,K.C. 2016, ApJ, 817, 84

Kregel, M., van der Kruit, P. C., \& Freeman, K. C. 2005, MNRAS, 358, 503

Kregel, M. \& van der Kruit, P. C. 2005, MNRAS, 358, 481

Kurapati, S., Banerjee, A., Chengalur, J. N., et al. 2018, MNRAS, 479, 5686

Kuzio de Naray, R. \& Kaufmann, T. 2011, MNRAS, 414, 3617

Leggett, S. K., Currie, M. J., Varricatt, W. P., Hawarden, T. G. et al. 2006, MNRAS, 373, 781

Maccio, A., Dutton, A., van den Bosch, F., et al. 2007, MNRAS, 378, 55

MacLachlan, J. M., Matthews, L. D., Wood, K., \& Gallagher, J. S. 2011, ApJ, 741, 6

Makarov, D. I., Burenkov, A. N., \& Tyurina, N. V. 2001, Astron. Letters, 27, 213

Makarov D., Prugniel P., Terekhova N., Courtois H., \& Vauglin I. 2014, A\&A, 570, A13

Matthews, L. D., Gallagher, J. S., \& van Driel, W. 1999, AJ, 118, 2751

Matthews, L.D., 2000, AJ, 120, 1764

Matthews, L. D. \& van Driel, W. 2000, A\&A, 143, 421

Matthews, L. D. \& Wood, K. 2001, ApJ, 548, 150

Matthews, L. D. \& Uson, J. M. 2008, AJ, 135, 291

Matthews, L. D. \& Uson, J. M. 2008b, ApJ, 688, 237

Mendelowitz, C. M., Matthews, L. D., Hibbard, J. E. \& Wilcots, E. M. BAAS, 197th AAS Meeting, 32,1459

Mitronova, S. N., Huchtmeier, W. K., Karachentsev, I. D., Karachentseva, V. E., et al. 2005, Astron. Letters, 31, 501

Mo, H. J., McGaugh, S. S., \& Bothun, G. D. 1994, MNRAS, 267, 129

Mogotsi, K. M.; de Blok, W. J. G.; Caldú-Primo, A. et al. 2016, AJ, 151,15

Mosenkov, A.V., Sotnikova, N.Ya., \& Reshetnikov, V.P. 2010, MNRAS, 401, 559

Mosenkov, A.V., Sotnikova, N.Ya., Reshetnikov, V.P., et al., 2015, MNRAS, 451, 2376

Naab, T. \& Burkert, A. 2003, ApJ, 597, 893

Narayan C. A. \& Jog C. J., 2002, A\&A, 394, 89

Narayan C. A., Saha K., Jog C. J., 2005, A\&A, 440, 523

O'Brien, J. C., Freeman, K. C. \& van der Kruit, P. C. 2010, A\&A, 515, 62

O'Neil, K., Bothun, G. D., \& Schombert, J. 2000, AJ, 119, 136

Peebles, P. J. E. 1969, ApJ, 155, 393

Pérez-Montaño, L. E. \& Cervantes Sodi, B. 2019, MNRAS, 490, 3773 
Phillipps, S., Edmunds, M. G., \& Davies, J. 1990, MNRAS, 244, 168

Polyachenko, V. L. \& Shukhman, I. G. 1977, Astronomy Letters, 3, 134

Polyachenko, V., Polyachenko, E., \& Strel'nikov, A. 1997, Astron. Letters 23, 525

Purcell, C. W., Kazantzidis, S., \& Bullock, J. S. 2009, ApJ, 694, L98

Qu, Y., Di Matteo, P., Lehnert, M. D. et al. 2011, A\&A, 535, 5

Rafikov, R. 2001, MNRAS, 323, 445

Romeo, A. \& Falstad, N. 2013, MNRAS, 433, 1389

Rosenbaum, S. D. \& Bomans, D. J. 2004, A\&A, 422, 5

Rosenbaum, S. D. Krusch, E., Bomans, D. J. \& Dettmar, R.-J. 2009, A\&A, 504, 807

Safronov, V. S. 1960, Ann. d'Astrophysique, 23, 979

Saha, K. 2014, arXiv:1403.1711

Salo, H., Laurikainen, E., Laine, J. et al. 2015, ApJS, 219, 4

Salucci, P., Wilkinson, M. I., Walker, M. et al. 2012, MNRAS, 420, 2034

Schlegel, D.J., Finkbeiner, D.P. \& Davis, M., 1998, ApJ, 500,525

Skillman, E. D. 1987, Proc. of a conference at the California Institute of Technology, Pasadena, California, June 16-19, 1986. Ed. C. J. Lonsdale Persson, p. 263

Sofue, Y., Tutui, Y., Honma, M. et al. 1999, ApJ, 523, 136

Sotnikova, N. \& Rodionov, S. 2003, Astronomy Letters, 29, 321

Sotnikova, N. \& Rodionov, S. 2005, Astronomy Letters, 31, 17

Sotnikova, N. \& Rodionov, S. 2006, Astronomy Letters, 32, 649

Spitzer, L., 1942, ApJ, 95, 329
Springob, C. M., Haynes, M. P., Giovanelli, R. \& Kent, B. R. 2005, ApJS, 160, 149

Toomre, A. 1964, ApJ, 139, 1217

Uson, J. \& Matthews, L. 2003, AJ,125, 2455

van Albada, T. S. \& Sancisi, R. 1986, Phil. Trans. R. Soc. London, A. 320, 447

van der Hulst, J. M., Skillman, E. D., Kennicutt, R. C., \& Bothun, G. D. 1987, A\&A, 177, 63

vad der Kruit, P. C. \& Searle, L. 1981, A\&A, 95, 105

vad der Kruit, P. C. \& Searle, L. 1982, A\&A, 110, 79

vad der Kruit, P. C. 1988, A\&A, 192, 117

van der Kruit, P. C., Jimenez-Vicente, J., Kregel, M., \&

Freeman, K. C. 2001, A\&A, 379, 374

Varela, J., Betancort-Rijo, J., Trujillo, I., \& Ricciardelli, E. 2012, ApJ, 744, 82

Velazquez, H. \& White, S. D. M. 1999, MNRAS, 304, 254

Vorobyov, E.I, Shchekinov, Yu., Bizyaev, D., Bomans, D. et al. 2009, A\&A, 505, 483

Vorontsov-Velyaminov, B. 1967, in Modern Astrophysics, ed. M. Hack (Paris: Gauthier-Villars), p.347

Walker, I. R., Mihos, J. C., \& Hernquist, L. 1996, ApJ, 460, 121

Wright, E. L., Eisenhardt, P. R. M., Mainzer, A. K. et al. 2010, AJ, 140, 1868

Xilouris, E. M., Byun, Y. I., Kylafis, N. D., et al. 1999, A\&A, 344, 868

Yoachim, P. \& Dalcanton, J. J. 2006, AJ, 131, 226

Yoachim, P. \& Dalcanton, J. J. 2008, ApJ, 682, 1004

Zasov, A.V., Makarov, D. I., \& Mikhailova, E. A. 1991, Astronomy Letters, 17, 374

Zasov, A.V., Bizyaev, D.V., Makarov, D. I., \& Tiurina, N.V., Astronomy Letters, 2002, 28, 527

Zasov, A. V. \& Khoperskov, A. V., 2003, Astronomy Letters, 29, 437 